\title{
Structural and Functional Synaptic Plasticity Induced by Convergent Synapse Loss in the Drosophila Neuromuscular Circuit
}

\author{
${ }^{\circledR}$ Yupu Wang, ${ }^{1,2,3}{ }^{\circledR}$ Meike Lobb-Rabe, ${ }^{1,2,4}{ }^{\oplus}$ James Ashley, ${ }^{1,2}$ Veera Anand, ${ }^{1,2}$ and ${ }^{\circledR}$ Robert A. Carrillo ${ }^{1,2,3,4}$ \\ ${ }^{1}$ Department of Molecular Genetics \& Cellular Biology, University of Chicago, Chicago, Illinois 60637, ${ }^{2}$ Grossman Institute for Neuroscience, Quantitative \\ Biology and Human Behavior, University of Chicago, Chicago, Illinois 60637, ${ }^{3}$ Committee on Development, Regeneration, and Stem Cell Biology, \\ University of Chicago, Chicago, Illinois 60637, and ${ }^{4}$ Program in Cell and Molecular Biology, University of Chicago, Chicago, Illinois 60637
}

Throughout the nervous system, the convergence of two or more presynaptic inputs on a target cell is commonly observed. The question we ask here is to what extent converging inputs influence each other's structural and functional synaptic plasticity. In complex circuits, isolating individual inputs is difficult because postsynaptic cells can receive thousands of inputs. An ideal model to address this question is the Drosophila larval neuromuscular junction (NMJ) where each postsynaptic muscle cell receives inputs from two glutamatergic types of motor neurons (MNs), known as $1 \mathrm{~b}$ and 1s MNs. Notably, each muscle is unique and receives input from a different combination of $1 \mathrm{~b}$ and 1s MNs; we surveyed multiple muscles for this reason. Here, we identified a cell-specific promoter that allows ablation of 1s MNs postinnervation and measured structural and functional responses of convergent $1 \mathrm{~b}$ NMJs using microscopy and electrophysiology. For all muscles examined in both sexes, ablation of $1 \mathrm{~s}$ MNs resulted in NMJ expansion and increased spontaneous neurotransmitter release at corresponding $1 \mathrm{~b}$ NMJs. This demonstrates that $1 \mathrm{~b}$ NMJs can compensate for the loss of convergent 1 s MNs. However, only a subset of $1 \mathrm{~b}$ NMJs showed compensatory evoked neurotransmission, suggesting target-specific plasticity. Silencing 1s MNs led to similar plasticity at $1 \mathrm{~b}$ NMJs, suggesting that evoked neurotransmission from $1 \mathrm{~s}$ MNs contributes to $1 \mathrm{~b}$ synaptic plasticity. Finally, we genetically blocked $1 \mathrm{~s}$ innervation in male larvae and robust $1 \mathrm{~b}$ synaptic plasticity was eliminated, raising the possibility that 1s NMJ formation is required to set up a reference for subsequent synaptic perturbations.

Key words: co-innervation; compensation; neuromuscular junction; neurotransmission; plasticity; synaptic growth

Significance Statement

In complex neural circuits, multiple convergent inputs contribute to the activity of the target cell, but whether synaptic plasticity exists among these inputs has not been thoroughly explored. In this study, we examined synaptic plasticity in the structurally and functionally tractable Drosophila larval neuromuscular system. In this convergent circuit, each muscle is innervated by a unique pair of motor neurons. Removal of one neuron after innervation causes the adjacent neuron to increase neuromuscular junction outgrowth and functional output. However, this is not a general feature as each motor neuron differentially compensates. Further, robust compensation requires initial coinnervation by both neurons. Understanding how neurons respond to perturbations in adjacent neurons will provide insight into nervous system plasticity in both healthy and disease states.

Received June 11, 2020; revised Nov. 28, 2020; accepted Dec. 17, 2020.

Author contributions: Y.W., J.A., and R.A.C. designed research; Y.W., M.L.-R., J.A., and V.A. performed research; Y.W., M.L.-R., J.A., and R.A.C. analyzed data; Y.W., J.A., and R.A.C. wrote the paper.

Stocks obtained from the Bloomington Drosophila Stock Center [National Institutes of Health (NIH) Grant P40-0D-018537] were used in this study. The monoclonal antibodies 3C10,4F3, and 8B4D2 were deposited to the Developmental Studies Hybridoma Bank (DSHB) by C. Goodman, while the nc82 antibody was deposited by E. Buchner and were obtained from the DSHB, created by the Eunice Kennedy Shriver National Institute of Child Health and Development of the NIH and maintained at The University of lowa, Department of Biology (lowa City, IA). This work was supported by NIH Grants K01-NS-102342 (to R.A.C.) and T32-GM-007183 (to M. L.-R.), funding from the University of Chicago Biological Sciences Division (BSD) International Student
Fellowship (to Y.W.), and funding from the BSD Office of Diversity and Inclusion (to R.A.C.) and the Grossman Institute for Neuroscience, Quantitative Biology and Human Behavior (to R.A.C.). We thank Edwin "Chip" Ferguson, Ellie Heckscher, Kai Zinn, Kai Wang, Hong Liu, Richard Fehon, David Pincus, and members of the Carrillo laboratory for helpful discussions and comments. We also thank Vytas Bindokas for helpful guidance for Imaris analysis.

The authors declare no competing financial interests.

Correspondence should be addressed to Robert A. Carrillo at robertcarrillo@uchicago.edu.

https://doi.org/10.1523/JNEUROSCl.1492-20.2020

Copyright $\odot 2021$ the authors 
A
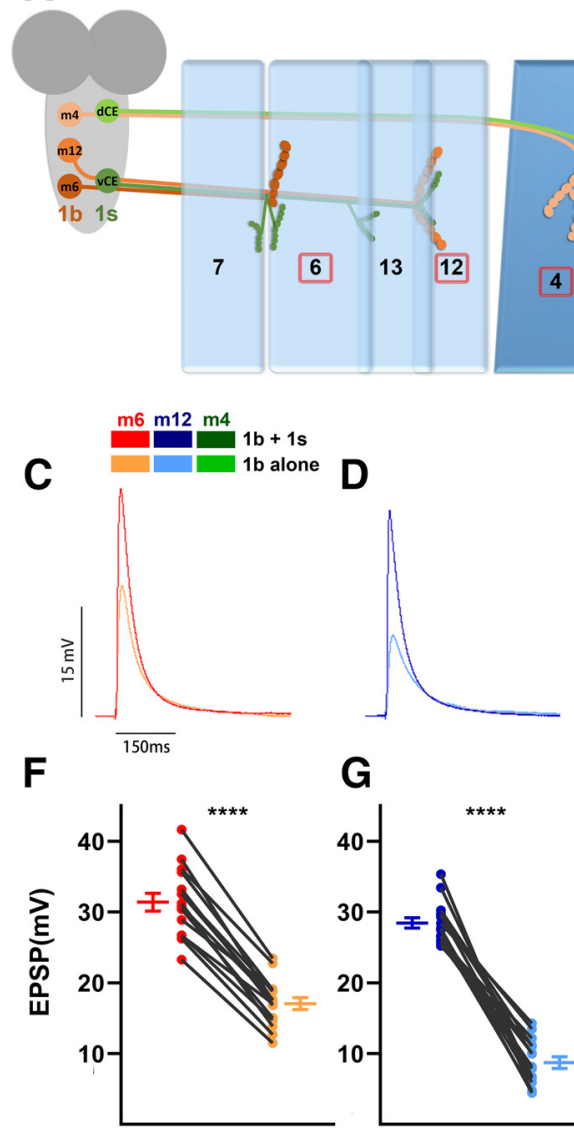

G

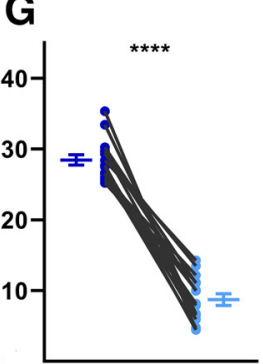

B

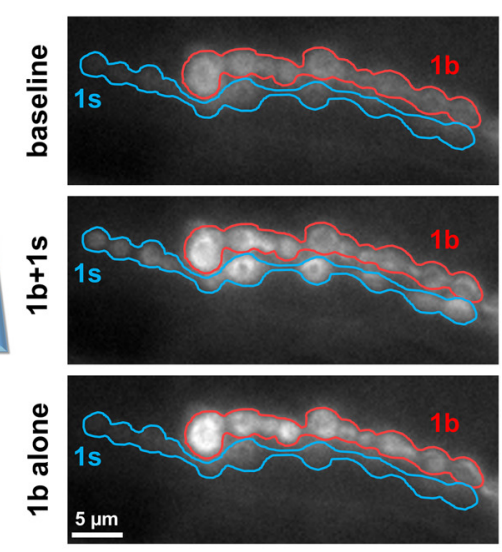

I

H
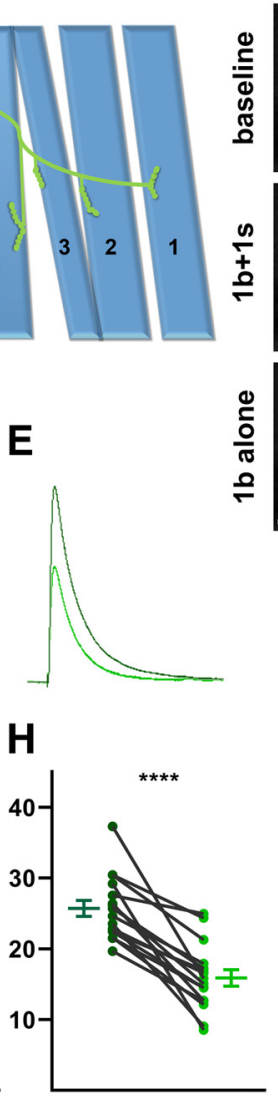

E
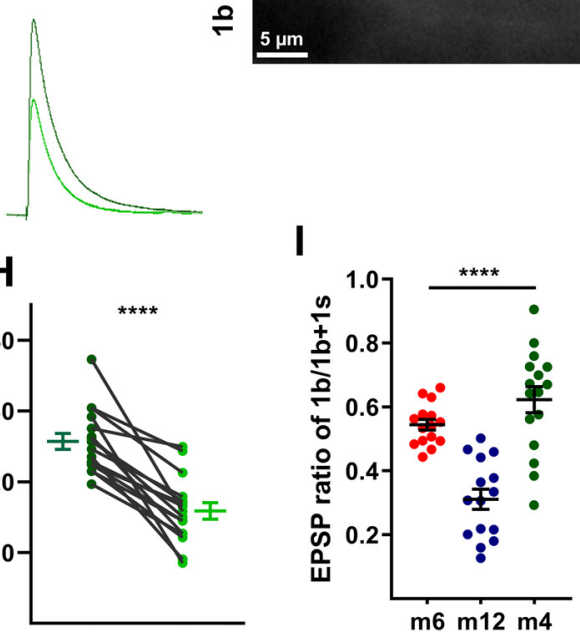

Figure 1. $1 \mathrm{~b}$ and $1 \mathrm{~s}$ MNs differentially contribute to the total EPSPS. $\boldsymbol{A}$, Schematic of the innervation pattern of a subset of 1s MNs (vCE: dark green; dCE: light green) and 1b MNs (m6-1b: rust; $\mathrm{m} 12$-1b: orange; $\mathrm{m} 4-1 \mathrm{~b}$ : peach). Muscles analyzed in this study are marked by the red boxes. $\boldsymbol{B}$, Representative frames showing the baseline fluorescence (top), $1 \mathrm{~b}+1 \mathrm{~s}$ firing event (middle), and $1 \mathrm{~b}$ alone firing event (bottom) of $\mathrm{m} 6$ in MHC-CD8::GCaMP6f-Sh larvae (1b: red; 1s: blue; also see Movie 1). C-E, Representative EPSP traces of $1 \mathrm{~b}+1 \mathrm{~s}$ and $1 \mathrm{~b}$ alone on $\mathrm{m} 6$ (C), $\mathrm{m} 12$ $(\boldsymbol{D})$, and $\mathrm{m} 4(\boldsymbol{E})$. Traces and graphs are color coded as indicated in the color key. $\boldsymbol{F}-\boldsymbol{H}$, Paired EPSP amplitudes of $\mathrm{m} 6\left(\boldsymbol{F}, t_{(14)}=18.60, p<0.0001\right.$, paired $t$ test $), \mathrm{m} 12\left(\boldsymbol{G}, t_{(14)}=15.73\right.$, $p<0.0001$, paired $t$ test), and $\mathrm{m} 4\left(\boldsymbol{H}, t_{(15)}=7.43, p<0.0001\right.$, paired $t$ test). $\boldsymbol{I}$, Calculated EPSP ratios of $1 \mathrm{~b} / 1 \mathrm{~b}+1 \mathrm{~s}$ of $\mathrm{m} 6$, $\mathrm{m} 12$, and $\mathrm{m} 4\left(F_{(2,43)}=26.03, p<0.0001\right.$, one-way ANOVA, Tukey post hoc test). Error bars indicate \pm SEM $* * * * p<0.0001$. $n$ values (NMJs/larva) are $15 / 12,15 / 12$, and $16 / 15$, respectively.

\section{Introduction}

The nervous system is characterized by complex wiring patterns that include different neurons converging onto the same postsynaptic cell. This wiring paradigm is found in pyramidal neurons that receive input from excitatory and inhibitory contacts (Megías et al., 2001), and in esophageal striated muscles that receive enteric and vagal nerve inputs (Kallmünzer et al., 2008; Neuhuber and Wörl, 2016). While dynamic regulation of individual synapses has been examined (Berry and Nedivi, 2017; Kruijssen and Wierenga, 2019), interplay between convergent neurons has been predominantly studied by monitoring postsynaptic spine changes (Oh et al., 2015; Hedrick et al., 2016; Jungenitz et al., 2018; Chistiakova et al., 2019). Understanding how convergent neurons respond to perturbations will shed light on the etiologies of neurodegenerative disorders, such as amyotrophic lateral sclerosis, which display progressive neuronal cell death and devastating functional consequences (Cluskey and Ramsden, 2001).

Analysis of individual inputs in the CNS is complicated by the high density of converging inputs on the same target cell. The Drosophila larval neuromuscular circuit, however, circumvents this with a simple, hard-wired connectivity map (Fig. 1A). The body plan is segmentally repeated, and each hemisegment is bilaterally symmetrical and composed of 35 motor neurons (MNs) and 30 postsynaptic muscles. Like most vertebrate central synapses, the individual glutamatergic neuromuscular contacts consist of axon terminal swellings, called boutons, and elaborate postsynaptic complexes (Collins and DiAntonio, 2007; Van Vactor and Sigrist, 2017). Each bouton houses several active zones (AZs) that enable neurotransmission. Larval muscles are innervated by two main MNs, type $1 \mathrm{~b}$ (big) and 1s (small), which resemble tonic and phasic neurons, respectively (Schaefer et al., 2010), unlike vertebrate skeletal muscles that are monoinnervated. Most $1 \mathrm{~b}$ MNs innervate a single muscle, whereas 1s MNs innervate functional muscle groups (Landgraf et al., 1997; Lnenicka and Keshishian, 2000; Hoang and Chiba, 2001), highlighting important structural and functional distinctions between these classes of neurons.

In the larval neuromuscular circuit, $1 \mathrm{~b}$ and $1 \mathrm{~s} \mathrm{MNs}$ are required for normal locomotion. Each MN type has unique electrophysiological properties: $1 \mathrm{~b}$ MNs display a higher rate of spontaneous neurotransmitter release but the quantal size is smaller compared with 1s MNs (Nguyen and Stewart, 2016; Newman et al., 2017). In traditional neuromuscular junction (NMJ) electrophysiology experiments, EPSPs in muscles represent simultaneous stimulation of both neurons. However, several studies have isolated $1 \mathrm{~b}$-derived and $1 \mathrm{~b}+1 \mathrm{~s}$-derived EPSPs from different muscles (Table 1) and demonstrated that convergent $1 \mathrm{~b}$ and $1 \mathrm{~s}$ MNs do not equally contribute to the EPSP amplitude. Several forms of synaptic plasticity have been observed in the larval NMJ, 
Table 1. Comparative $1 \mathrm{~b}$ and 1s physiology from prior NMJ studies

\begin{tabular}{|c|c|c|c|c|c|}
\hline Muscle & $\begin{array}{l}\text { 1b EPSP } \\
(\mathrm{mV})\end{array}$ & $\begin{array}{l}\text { 1s EPSP } \\
\text { (mV) }\end{array}$ & $\begin{array}{l}1 \mathrm{~b}+1 \mathrm{~s} \text { EPSP } \\
(\mathrm{mV})\end{array}$ & {$\left[\mathrm{Ca}^{2+}\right]$} & Reference \\
\hline 1 & 11.8 & & 24.2 & $0.3 \mathrm{~mm}$ & $\begin{array}{l}\text { Aponte-Santiago et al., } \\
2020\end{array}$ \\
\hline 6 & 14.9 & & 24.3 & $1.0 \mathrm{~mm}$ & Li et al., 2002 \\
\hline 6 & $\sim 10$ & & $\sim 35$ & $1.8 \mathrm{~mm}$ & Kurdyak et al., 1994 \\
\hline 6 & 15.6 & 25.7 & & $0.5 \mathrm{~mm}$ & Genc and Davis, $2019^{a}$ \\
\hline 6 & 15.6 & & 23.1 & $1.5 \mathrm{~mm}$ & $\begin{array}{l}\text { Lnenicka and Keshishian, } \\
2000\end{array}$ \\
\hline 7 & 12.1 & & 15.5 & $1.5 \mathrm{~mm}$ & $\begin{array}{l}\text { Lnenicka and Keshishian, } \\
2000\end{array}$ \\
\hline
\end{tabular}

${ }^{\mathrm{a}}$ This study used optogenetics, instead of voltage injection, to differentially activate $1 \mathrm{~b}$ or $1 \mathrm{~s}$.

Table 2. Fly strains used in this study

\begin{tabular}{ll}
\hline Fly strain & Source \\
\hline$w^{1118}$ & Carrillo et al. (2015) \\
A8-GAL4 & Venkatasubramanian et al. (2019) \\
10XUAS-mCD8::GFP & Bloomington Drosophila Stock Center, stock \#32184 \\
10XUAS-mCD8::GFP,A8-GAL4 & This article \\
DIP- $\alpha^{\text {null }}$ & (Xu et al., 2018) \\
UAS-hid,rpr & Zhou et al. (1997) \\
UAS-TNT & Bloomington Drosophila Stock Center, stock \#28838 \\
MHC-CD8::GCaMP6f-Sh & Newman et al. (2017) \\
\hline
\end{tabular}

Table 3. Antibodies used in this study

\begin{tabular}{|c|c|c|}
\hline Antibody & Source & Concentration \\
\hline Rabbit anti-GFP & Thermo Fisher Scientific, catalog \#A11122 & $1: 500$ \\
\hline Chicken anti-GFP & Thermo Fisher Scientific, catalog \#A10262 & $1: 500$ \\
\hline Rabbit anti-Fas2 & Budnik Laboratory (Koh et al., 1999) & $1: 3500$ \\
\hline Rabbit anti-DLG & Budnik Laboratory (Koh et al., 1999) & $1: 40,000$ \\
\hline Mouse anti-DLG & $4 \mathrm{~F} 3, \mathrm{DSHB}$ & $1: 100$ \\
\hline Mouse anti-Eve & $3 C 10$, DSHB & $1: 50$ \\
\hline $\begin{array}{l}\text { Mouse anti-Bruchpilot } \\
\text { (BRP) }\end{array}$ & nc82, DSHB & $1: 50$ \\
\hline Mouse anti-GluRIIA & 8B4D2 (MH2B), DSHB & $1: 50$ \\
\hline Goat anti-HRP 405 & $\begin{array}{l}\text { Jackson ImmunoResearch, catalog \#123- } \\
\text { 475-021 }\end{array}$ & $1: 50$ \\
\hline $\begin{array}{l}\text { Goat anti-HRP-Alexa Fluor } \\
\quad 647\end{array}$ & $\begin{array}{l}\text { Jackson ImmunoResearch, catalog \#123- } \\
\quad 605-021\end{array}$ & $1: 50$ \\
\hline $\begin{array}{l}\text { Goat anti-mouse-Alexa } \\
\text { Fluor } 568\end{array}$ & Thermo Fisher Scientific, catalog \#A11031 & $1: 500$ \\
\hline $\begin{array}{l}\text { Goat anti-chicken-Alexa } \\
\text { Fluor } 488\end{array}$ & Thermo Fisher Scientific, catalog \#A11039 & $1: 500$ \\
\hline $\begin{array}{l}\text { Goat anti-rabbit-Alexa } \\
\text { Fluor } 405\end{array}$ & Thermo Fisher Scientific, catalog \#A31556 & $1: 500$ \\
\hline $\begin{array}{l}\text { Goat anti-rabbit-Alexa } \\
\text { Fluor } 568\end{array}$ & Thermo Fisher Scientific, catalog \#A11036 & $1: 500$ \\
\hline $\begin{array}{l}\text { Goat anti-rabbit-Alexa } \\
\text { Fluor } 647\end{array}$ & Thermo Fisher Scientific, catalog \#A32733 & $1: 500$ \\
\hline
\end{tabular}

including facilitation and depression (Hallermann et al., 2010). As each muscle is coinnervated and individual synaptic activities can be distinguished, this system provides an ideal platform to investigate structural and physiological plasticity changes that enable one $\mathrm{MN}$ to compensate for perturbations of a convergent $\mathrm{MN}$. A recent study found that ablating the $1 \mathrm{~s} \mathrm{MN}$ on muscle 1 (m1) elevated evoked neurotransmission of the corresponding $1 \mathrm{~b}$ NMJ (Aponte-Santiago et al., 2020). However, each muscle is innervated by a unique $1 \mathrm{~b}-1 \mathrm{~s} \mathrm{MN}$ pair, so it is unclear whether this plasticity is consistent across all muscles.
Table 4. Recorded resting potentials

\begin{tabular}{|c|c|}
\hline & Resting potential $(-\mathrm{mV})$ \\
\hline \multicolumn{2}{|l|}{ Figure 1} \\
\hline $\begin{array}{l}m 6,1 b+1 s \\
m 6,1 b\end{array}$ & $65.31 \pm 3.119$ \\
\hline $\begin{array}{l}\mathrm{m} 121 \mathrm{~b}+1 \mathrm{~s} \\
\mathrm{~m} 12,1 \mathrm{~b}\end{array}$ & $62.60 \pm 3.942$ \\
\hline $\begin{array}{l}m 4,1 b+1 s \\
m 4,1 b\end{array}$ & $61.50 \pm 3.778$ \\
\hline \multicolumn{2}{|l|}{ Figure 6} \\
\hline $\mathrm{m} 6, A 8>G F P$ & $63.46 \pm 2.824$ \\
\hline $\mathrm{m6}, A 8>\mathrm{GFP}$,hid,rpr & $67.03 \pm 3.247$ \\
\hline $\mathrm{m} 12, A 8>G F P$ & $64.13 \pm 2.586$ \\
\hline $\mathrm{m} 12$, A8> GFP, hid, rpr & $63.71 \pm 4.947$ \\
\hline $\mathrm{m} 4, A 8>G F P$ & $63.32 \pm 2.933$ \\
\hline $\mathrm{m} 4$, A8 $>$ GFP, hid,rpr & $64.76 \pm 2.985$ \\
\hline \multicolumn{2}{|l|}{ Figure 10} \\
\hline$A 8>G F P$ & $63.67 \pm 1.732$ \\
\hline$A 8>G F P, T N T$ & $64.56 \pm 3.046$ \\
\hline \multicolumn{2}{|l|}{ Figure 11} \\
\hline$A 8>G F P$ & $62.07 \pm 2.154$ \\
\hline DIP-alpha ${ }^{\text {null }}, A 8>G F P$ & $62.62 \pm 3.160$ \\
\hline \multicolumn{2}{|l|}{ Figure 12} \\
\hline $\mathrm{m} 4$ with $1 \mathrm{~s}$ & $62.70 \pm 2.263$ \\
\hline m4 with no 1s & $62.30 \pm 3.310$ \\
\hline
\end{tabular}

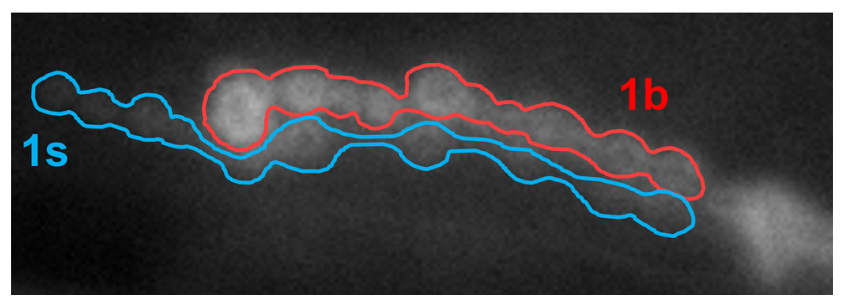

Movie 1. SynapGCaMP allows $1 \mathrm{~b}$ and $1 \mathrm{~b}+1 \mathrm{~s}$-derived EPSPs from larval muscles to be differentiated. Nerves were stimulated at $0.5 \mathrm{~Hz}$ using different voltages for the two stimulus conditions. Frame rate is 30. [View online]

In this study, we examined three separate muscles to test structural and functional plasticity. First, we confirmed musclespecific $1 \mathrm{~b}$ activities. Next, we ablated 1s MNs after innervation and examined corresponding $1 \mathrm{~b}$ NMJ compensatory responses. We found that all three $1 \mathrm{~b}$ NMJs increased their bouton numbers and rates of spontaneous neurotransmitter release without a corresponding upregulation of AZs and some $1 \mathrm{~b}$ NMJs also partially increased their evoked neurotransmission. An important factor for $1 \mathrm{~b}$ synaptic plasticity is the $1 \mathrm{~s}$ EPSP as silencing 1 s evoked activity caused similar $1 \mathrm{~b} \mathrm{NMJ}$ responses. Additionally, robust $1 \mathrm{~b}$ synaptic plasticity does not occur if the $1 \mathrm{~s} \mathrm{NMJ}$ is never formed, indicating that initial $1 \mathrm{~s}$ innervation is required. These results demonstrate a novel, target-specific synaptic plasticity that may be a common feature of convergent neural circuits.

\section{Materials and Methods}

Fly and antibody reagents

Fly lines and antibodies used in this study are listed in Tables 2 and 3.

Dissection and immunocytochemistry

Embryonic dissections were performed as previously described (Lee et al., 2009). Egg-laying chambers were set up with adult flies (15-20 females and 10-15 males) and capped with grape juice plates (3\% agar, $1.3 \%$ sucrose, $25 \%$ grape juice in water). After $6 \mathrm{~h}$ laying periods, grape 

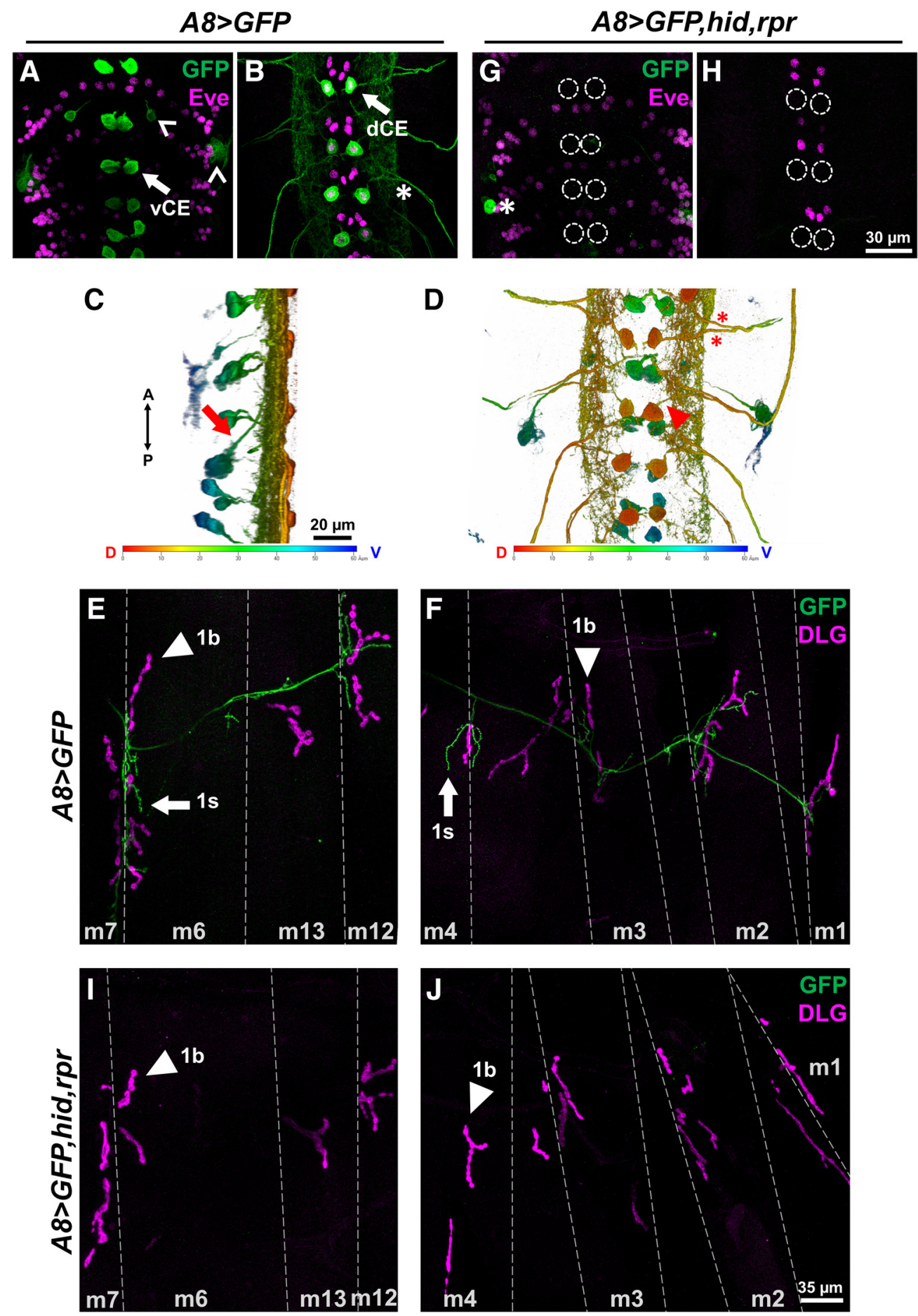

Figure 2. A8-GAL4 drives expression in 1s MNs and can be used to ablate 1s MNs. $\boldsymbol{A}, \boldsymbol{B}$, Representative third instar larval VNC $\boldsymbol{Z}$-sections showing ventral $(\boldsymbol{A})$ and dorsal $(\boldsymbol{B})$ cell bodies labeled with GFP (green) and Eve (magenta; labels nuclei of dCE and other neurons) in A8> GFP. Arrows indicate GFP-positive vCE and dCE cell bodies. Carets indicate other cells that express $A 8$. Asterisks indicate two axons exiting the VNC. C, $\boldsymbol{D}, 3 \mathrm{D}$ representations of $A 8$-expressing neurons in the VNC viewed from lateral $(\boldsymbol{C})$ and dorsal $(\boldsymbol{D})$ sides (same VNC as $\boldsymbol{A}$ and $\boldsymbol{B}$ ). Arrow in $\boldsymbol{C}$ shows a ventral cell (left, green) that projects an axon to the dorsal midline. Arrowhead in $\boldsymbol{D}$ shows an ipsilateral projection from a dorsal cell. Heat map colors are as follows: red denotes the dorsal-most region; and blue denotes the ventral most region. Asterisks indicate axons exiting the VNC similar to $\boldsymbol{B}$. $\boldsymbol{E}, \boldsymbol{F}$, Representative third instar larval abdominal hemisegment labeled with GFP (green) and the postsynaptic marker DLG (magenta) in A8> GFP. Ventral muscles $(\mathrm{m} 6, \mathrm{~m} 7, \mathrm{~m} 13$, and $\mathrm{m} 12)$ innervated by VCE $(\boldsymbol{E})$ and dorsal muscles $(\mathrm{m} 4, \mathrm{~m} 3, \mathrm{~m} 2$ and $\mathrm{m} 1)$ innervated by dCE $(\boldsymbol{F})$. Arrows indicate $1 \mathrm{~s}$ NMJs and arrowheads indicate $1 \mathrm{~b}$ NMJs. $\boldsymbol{G}, \boldsymbol{H}$, Representative third instar larval VNCS lacking vCE $(\boldsymbol{G})$ and dCE $(\boldsymbol{H})$ labeled with GFP (green) and Eve (magenta) in A8> GFP,hid,rpr. Dashed circles mark the absence of vCE and dCE. Note that both vCE and dCE cell bodies are ablated by the third instar stage. Asterisk marks a GFP-positive cell that remained. I, J, Representative third instar larval abdominal hemisegment showing ventral muscles ( $)$ and dorsal muscles (Л), labeled with GFP (green) and DLG (magenta) in A8> GFP,hid,rpr. Note that all NMJs from vCE and dCE are absent (no GFP). Arrowheads indicate $1 \mathrm{~b}$ NMJs.

plates covered in embryos were collected. Embryos were staged on double-sided tape using the autofluorescence and shape of the gut (Bownes, 1975; Hartenstein et al., 1987) under a Zeiss V20 stereoscope, and then dechorionated with a sharpened metal probe and placed on grape juice agar. Embryos were transferred to double-sided tape on a Superfrost
Plus slides (catalog \#22-037-246, Thermo Fisher Scientific) with the dissecting area outlined by a PAP pen (catalog \#195506, Research Products International), and then covered with $0.22 \mu \mathrm{m}$ filtered PBS $(0.01 \mathrm{M}$ sodium phosphate, $150 \mathrm{~mm}$ sodium chloride). Embryos were opened with an electrolytically sharpened tungsten wire, removed from the 

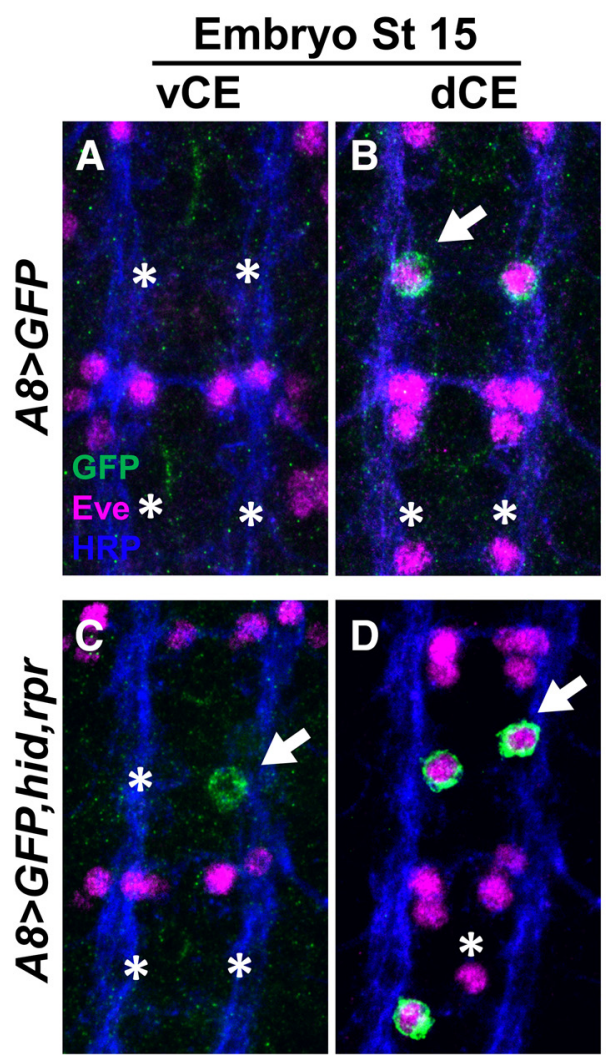

\section{Early $1^{\text {st }}$ instar}
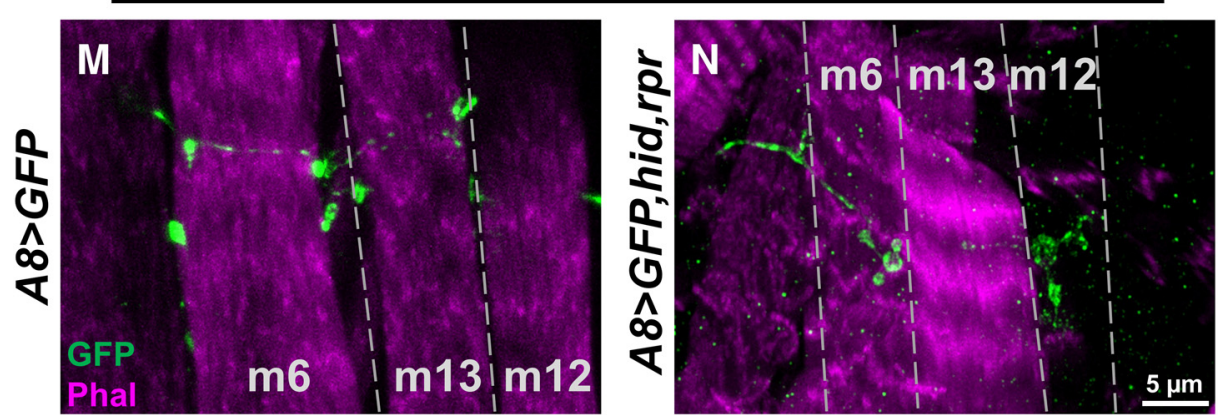

O Innervation frequency (\%)

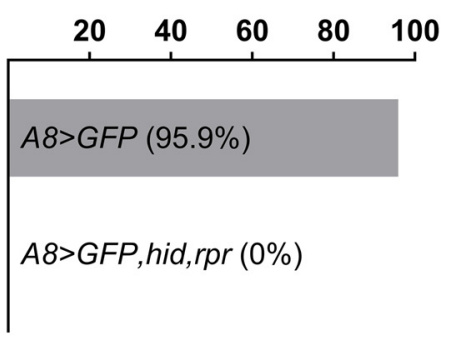

Figure 3. A8>hid,rpr-induced cell death occurs after 1s innervation. $\boldsymbol{A}-\boldsymbol{L}$, Representative images depicting the presence or absence of $v C E$ and dCE cell bodies from embryonic stage 15 ( $\boldsymbol{A}-$ D), stage $17(\boldsymbol{E}-\boldsymbol{H})$, and early first instar $(\boldsymbol{I}-\boldsymbol{L})$ larval VNCs labeled with GFP (green), Eve (magenta), and Fasciclin 2 (blue) in control (A8>GFP) and 1s-ablated (A8>GFP,hid,rpr) animals. Arrows and asterisks indicate the cells expressing or not expressing $A 8$, respectively. $\boldsymbol{A}, \boldsymbol{B}, A 8$ expression begins at embryonic stage 15 . In $A 8>$ GFP, hid,rpr, VCEs, and dCEs undergo apoptosis starting at embryonic stage $17(\boldsymbol{G}, \boldsymbol{H})$, noted by the loss of Eve staining in dCE and GFP-positive debris (indicated by arrowhead), and are completely absent in early first instar larvae $(\boldsymbol{K}, \boldsymbol{L})$. $\boldsymbol{M}$, $\boldsymbol{N}$, Representative 1s NMJs labeled with GFP (green) and a muscle marker, phalloidin (magenta), in control $(\boldsymbol{M})$ and 1s-ablated early first instar larvae $(\boldsymbol{N})$. Note that 1s NMJs are labeled by GFP in control animals, and some 1s NMJs are still present in A8>GFP,hid,rpr animals, suggesting that A8>GFP,hid,rpr-induced cell death happens after 1s NMJ formation. $\mathbf{0}$, Innervation frequency of $1 \mathrm{~s}$ MNs in control and 1s-ablated late first instar larvae. Three muscles ( $\mathrm{m} 6, \mathrm{~m} 12$, and $\mathrm{m} 4$ ) were pooled and analyzed together. All 1s NMJs were eliminated in 1s-ablated animals by this stage. $n$ values (NMJs/larva) are $76 / 5$ and $86 / 5$ for $\boldsymbol{0}$.

vitellin membrane, and then adhered to the charged slide. Dissected embryos were washed once with PBS, and then fixed for $30 \mathrm{~min}$ at room temperature using 4\% paraformaldehyde (Electron Microscopy Sciences). Samples were then washed three times in $0.05 \%$ PBST (PBS with $0.05 \%$ Triton $\mathrm{X}-100)$, and then blocked for $1 \mathrm{~h}$ in $5 \%$ normal goat serum ( $5 \%$ goat serum diluted in $0.05 \%$ PBST). Samples were incubated in primary antibody solutions overnight at $4^{\circ} \mathrm{C}$ and washed three times in PBST. Samples were then incubated with secondary antibody solutions at room temperature for $2 \mathrm{~h}$ and washed three times with PBST. Finally, samples were mounted in Vectashield (Vector Laboratories), and the coverslip was sealed with nail polish.

First and third instar larval dissections were performed as previously described (Ashley et al., 2019). Wandering third instar larvae were dissected in PBS on Sylgard-184 (Dow) dishes and pinned down using sharpened $0.1 \mathrm{~mm}$ insect pins (catalog \#26002-10, Fine Science Tools). For first instar larvae, electrolytically sharpened tungsten pins were used to accommodate the size of smaller larvae. Samples were then fixed for $30 \mathrm{~min}$ using $4 \%$ paraformaldehyde [or $5 \mathrm{~min}$ in Bouin's solution for glutamate receptor IIA (GluRIIA)] and then transferred to 0.5 $\mathrm{ml}$ Eppendorf tubes. Samples were blocked and treated with primary and secondary antibodies as the embryo samples described above. All larval washes and antibody incubations were performed with mild agitation on a nutator.

\section{Image acquisition}

All imaging was acquired on a Zeiss LSM800 confocal microscope with either a $20 \times$ plan-apo 0.8 numerical aperture (NA) objective, a $40 \times$ planneofluar $1.3 \mathrm{NA}$ objective, or a $63 \times$ plan-apo $1.4 \mathrm{NA}$ objective. Laser 
intensity, pinhole, and gain were adjusted to increase the signal but avoid overexposure. All samples from the same experiment were imaged under identical conditions. Representative images are the maximum projection of the corresponding confocal $z$-stack (ImageJ).

\section{Image analysis}

Dorsal common exciter and ventral common exciter identification. The existence of ventral common exciter (vCE) and dorsal common exciter (dCE) MNs (two 1s type $\mathrm{MNs}$ ) was confirmed in embryos and first instars. dCEs were identified by the expression of GFP, the transcription factor Evenskipped (Eve), and their positions in the ventral nerve cord (VNC). vCEs were identified by the expression of GFP and their positions in the VNC. Final confirmation was done by identifying the muscle innervation patterns in larval abdominal hemisegments.

$1 b$ bouton counting. $1 \mathrm{~b}$ bouton counting was performed in third instar samples. Boutons were examined using HRP and scored as $1 \mathrm{~s}$ or $1 \mathrm{~b}$ based on the Discs large (DLG) signal, as $1 \mathrm{~s}$ boutons have a smaller and dimmer DLG signal than $1 \mathrm{~b}$ boutons (Guan et al., 1996). Satellite boutons were identified as small bud-like structures emerging from "parent" boutons (Lee and $\mathrm{Wu}, 2010)$.

Bruchpilot and GluRIIA quantification. Bruchpilot (BRP) and GluRIIA signals at $1 \mathrm{~b}$ NMJs were quantified in a manner similar to that described in the study by Han et al. (2020). Briefly, BRP and GluRIIA in 1s NMJs were excluded in controls by using the GFP signal $(A 8>G F P)$ to create a surface boundary (Imaris, Oxford Instruments) and setting the BRP and GluRIIA signal intensities inside 1s NMJs to zero. Next, a $1 b$ NMJ surface was created based on the masked BRP, or GluRIIA, channel, and the total intensities of horseradish peroxidase (HRP), DLG, and BRP or GluRIIA were collected. For normalization, A8> hid, $r \mathrm{pr}$ and control samples were normalized to corresponding mean control values. For BRP/HRP and GluRIIA/DLG ratios, each sample was normalized to the sum intensity of either HRP or DLG, respectively.

\section{Electrophysiology and analysis}

Current-clamp recordings were performed as previously described (Meng et al., 2020). Third instar larvae were dissected in modified HL3 saline (70 mм NaCl, $5 \mathrm{~mm} \mathrm{KCl,} 10 \mathrm{~mm} \mathrm{MgCl}$, 10 mм NaHCO3, $5 \mathrm{~mm}$ trehelose, $115 \mathrm{~mm}$ sucrose, $5 \mathrm{~mm}$ HEPES) with $0.3 \mathrm{~mm}$ calcium. Segmental nerves were cut near the ventral nerve cord to remove VNC input and then the larval fillet was perfused with modified HL3 saline containing $0.5 \mathrm{~mm}$ calcium. Body-wall muscle 6,12 , or 4 in abdominal segment 3 were impaled with a 15-30 $\mathrm{M} \Omega$ sharp electrode filled with $3 \mathrm{M} \mathrm{KCl}$ and recorded for miniature EPSPs (mEPSPs) for $2 \mathrm{~min}$. All mEPSP amplitudes from the same genotype were pooled together and binned with at $0.01 \mathrm{mV}$ increments to calculate the cumulative probability.

Nerves were drawn into a suction electrode and stimulated to elicit EPSPs. Specifically, for muscle 6 and 12 EPSP recordings, the whole segmental nerve bundle was stimulated, whereas for muscle 4 EPSP recording, the intersegmental nerve (ISN) above muscle 5 was stimulated. For each muscle, 24 EPSPs were elicited at $0.2 \mathrm{~Hz}$ and the largest 12 EPSPs were averaged to indicate the mean EPSP (while measuring EPSPs in A8>hid,rpr, we sometimes observed smaller EPSPs, similar to those m12
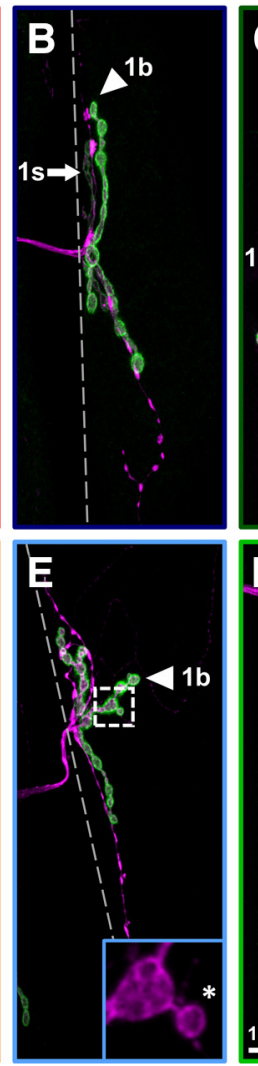

m4

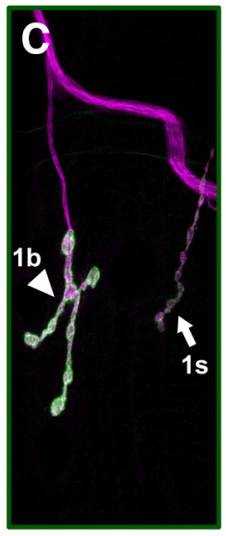

$\mathbf{F}$

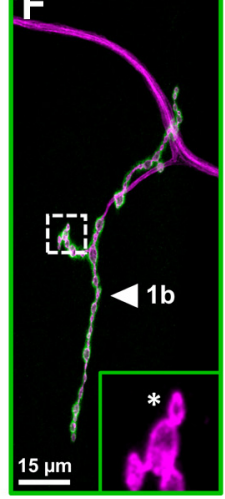

G

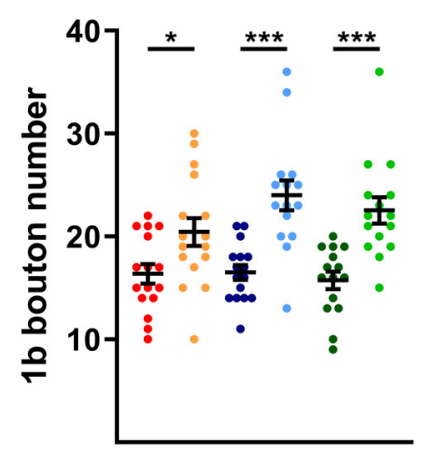

H

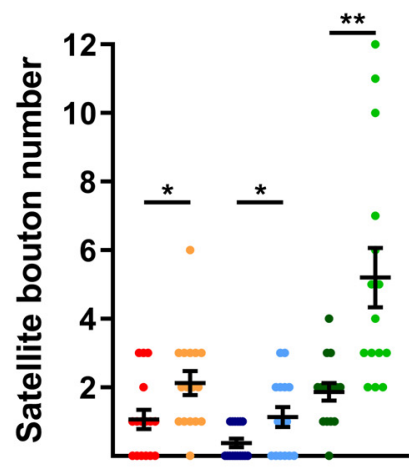

Figure 4. 1b NMJs expand upon ablation of $1 \mathrm{~s}$ MNs. $\boldsymbol{A}-\boldsymbol{F}$, Representative NMJ arbors (1b arbors and $1 \mathrm{~s}$ arbors, arrowheads ( unpaired $t$ test), $\mathrm{m} 12\left(t_{(19.05)}=2.397, p=0.0269\right.$, unpaired $t$ test with Welch's correction), and $\mathrm{m} 4\left(t_{(16.41)}=3.682, p=0.0019\right.$, unpaired $t$ test with Welch's correction) in control and 1s-ablated animals. Error bars indicate \pm SEM. $* p<0.05, * * p<0.01$, $* * * p<0.001 . n$ values (NMJs/larva) are 16/8, 16/8, 16/8, 15/8, 15/8, and 15/8, respectively.

reported by Lnenicka and Keshishian (2000)). All resting potentials are reported in Table 4.

Because of nonlinear summation of quantal units of large EPSPs, we calculated corrected EPSPs using the equation defined by Martin (1955) and elaborated by Feeney et al. (1998). Quantal content (QC) was then calculated by dividing the corrected mean EPSP amplitude by the mean mEPSP amplitude for each muscle. Specifically, in genotypes where $1 \mathrm{~b}$ and 1s NMJs were both present, the mean of the smaller two-thirds of mEPSP amplitudes was used to represent the $1 \mathrm{~b}$-derived mEPSP. This assumption was based on the published spontaneous frequencies of $1 \mathrm{~b}$ and 1s MNs (Newman et al., 2017). Then the 1b-derived EPSP was divided by the $1 \mathrm{~b}$-derived mEPSP amplitude to estimate the $1 \mathrm{~b}$-derived QC. Similarly, the $1 b+1 s$ EPSP was divided by the overall mEPSP amplitude to represent $1 \mathrm{~b}+1 \mathrm{~s}$ QC. Finally, the $1 \mathrm{~b}$-derived QC was normalized to the $1 \mathrm{~b}+1 \mathrm{~s}$ QC to indicate the estimated $1 \mathrm{~b}$ baseline QC (1b QC/ $1 \mathrm{~b}+1 \mathrm{~s} \mathrm{QC})$.

Paired-pulse recordings were performed under two-electrode voltageclamp configuration (Li et al., 2018) in modified HL3 saline with $0.5 \mathrm{~mm}$ calcium. A second sharp electrode with 10-15 $\mathrm{M} \Omega$ resistance was used to inject current. Muscle 6 was clamped at $-70 \mathrm{mV}$, and the nerve was stimulated by two pulses $20 \mathrm{~ms}$ apart at $0.1 \mathrm{~Hz}$. Only muscles with a leak current $<10 \mathrm{nA}$ were subjected to analysis. Paired-pulse ratios (EPSC2/ EPSC1) were calculated by dividing the second EPSC by the first EPSC.

Signals were amplified using a MultiClamp 700B amplifier (Molecular Devices) for EPSP and mEPSP recordings or a Geneclamp 
A

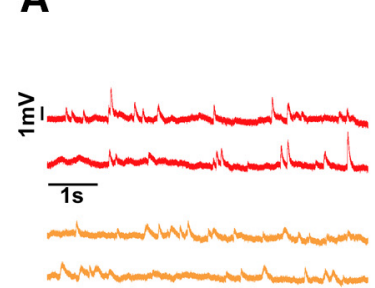

D

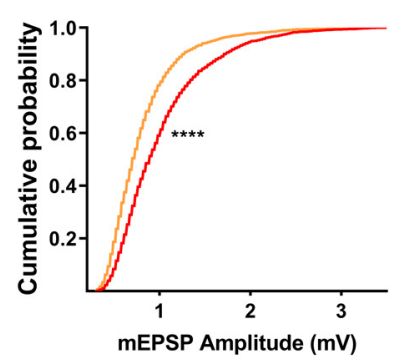

B

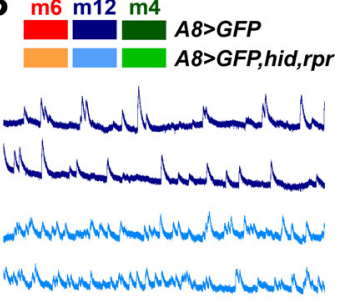

E

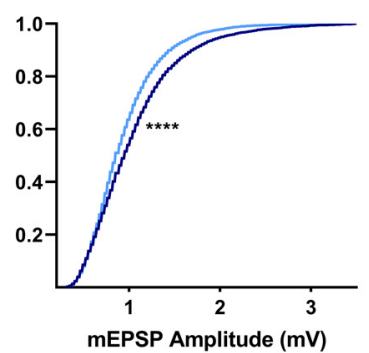

C

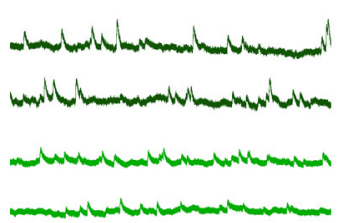

$\mathbf{F}$

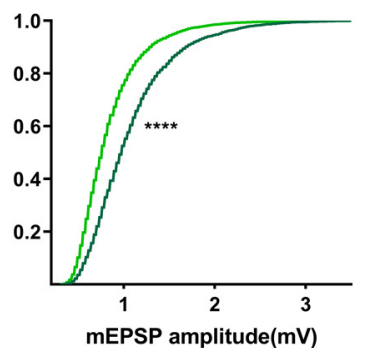

G

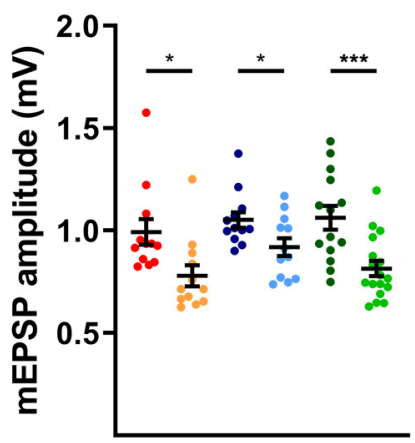

H

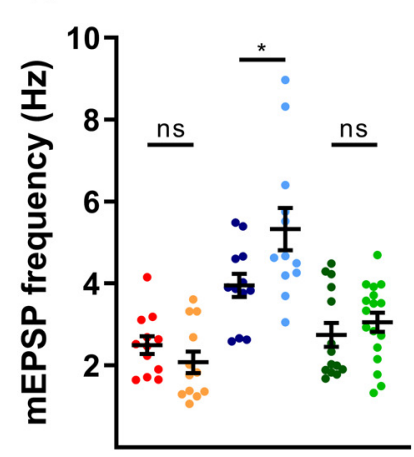

Figure 5. Loss of $1 \mathrm{~s}$ MNs decreases overall $m E P S P$ amplitudes and increases $1 \mathrm{~b} m E P S P$ frequencies. $A-C$, Representative mEPSP recordings of $\mathrm{m} 6(\boldsymbol{A}), \mathrm{m} 12(\boldsymbol{B})$, and $\mathrm{m} 4(\boldsymbol{C})$ in control $(A 8>$ GFP) and 1s-ablated (A8>GFP,hid,rpr) animals. Traces and graphs are color coded as indicated in the color key. $\boldsymbol{D}-\boldsymbol{F}$, Pooled cumulative probability distributions of $\mathrm{m} 6(\boldsymbol{D}$; $p<0.0001, \mathrm{~K}-\mathrm{S}$ test), $\mathrm{m} 12(\boldsymbol{E} ; p<0.0001, \mathrm{~K}-\mathrm{S}$ test), and $\mathrm{m} 4(\boldsymbol{F} ; p<0.0001$, K-S test). G, Quantification of mEPSP amplitude of $\mathrm{m} 6\left(t_{(22)}=2.630, p=0.0153\right.$, unpaired $t$ test), $\mathrm{m} 12 \quad\left(t_{(22)}=2.294, p=0.0317\right.$, unpaired $t$ test), and $\mathrm{m} 4$ $\left(t_{(29)}=3.700, p=0.0009\right.$, unpaired $t$ test) in control and 1s-ablated animals. Each data point represents the average mEPSP amplitude from one sample. $\boldsymbol{H}$, Quantification of mEPSP frequencies of $\mathrm{m} 6\left(t_{(22)}=1.224, p=0.2339\right.$, unpaired $t$ test), $\mathrm{m} 12$ $\left(t_{(22)}=2.331, p=0.0293\right.$, unpaired $t$ test $)$, and $\mathrm{m} 4\left(t_{(29)}=0.8369, p=0.4095\right.$, unpaired $t$ test) in control and 1s-ablated animals. Each data point represents the average mEPSP frequency from one sample. Error bars indicate \pm SEM. $* p<0.05$, $* * * p<0.001, * * * * p<0.0001$, ns - not significant. $n$ values (NMJs/larva) are 12/9, 12/11, 12/9, 12/12, 14/11, and 17/ 14 , respectively.

500B amplifier (Molecular Devices) for paired-pulse recordings and digitized with a Digidata 1550B (Molecular Devices). Stimulus was triggered via a Master-9 stimulator (A.M.P.I.). Data were acquired in pCLAMP 10 software (Molecular Devices) and analyzed using Mini Analysis software (Synaptosoft).

\section{GCaMP imaging coupled with electrophysiology}

Third instar MHC-CD8::GCaMP6f-Sh larvae were dissected in modified HL3 saline with $0.5 \mathrm{~mm}$ calcium and followed the same procedure of electrophysiology. Larval fillets were visualized using a Nikon FS microscope with a $40 \times$ long-working distance objective and GCaMP-positive $1 \mathrm{~b}$ and $1 \mathrm{~s}$ NMJs of muscles 6,12 , and 4 were illuminated by an Aura II solid-state illuminator. Together with electrophysiology stimulation, each stimulus triggered a GCaMP firing event with the corresponding EPSP. GCaMP signals were scanned and recorded by a PCO Edge 4.2 camera and NIS-Elements Imaging Software. Electrophysiology data were collected as described above. Given that $1 \mathrm{~b}$ MNs have a lower evoked threshold than 1s MNs, stimulating voltages were tuned to isolate
$1 \mathrm{~b}$ alone firing events and $1 \mathrm{~b}+1 \mathrm{~s}$ firing events (Movie 1). If a stimulation only triggered a GCaMP firing event at $1 \mathrm{~b}$ NMJs but not $1 \mathrm{~s}$ NMJs, the corresponding EPSP was counted as a $1 \mathrm{~b}$ alone EPSP. If a stimulation triggered GCaMP fluorescence changes at both $1 \mathrm{~b}$ and $1 \mathrm{~s}$ NMJs, the corresponding EPSP was counted as a $1 b+1$ s EPSP. For each sample, $1 \mathrm{~b}$ alone EPSPs and $1 \mathrm{~b}+1 \mathrm{~s}$ EPSPs were averaged respectively. $1 \mathrm{~b}$ alone/ $1 \mathrm{~b}+1 \mathrm{~s}$ was calculated by dividing the mean $1 \mathrm{~b}$ alone EPSP by the mean $1 b+1 s$ EPSP of each sample.

Experimental design and statistical analysis In all experiments, we included $A 8>G F P$ to ensure that 1s NMJs were present in control animals (Ashley et al., 2019; Aponte-Santiago et al., 2020). For experiments with $D I P-\alpha^{\text {null, }}$; $A 8>$ GFP larvae, males were selected as $D I P-\alpha$ on the X chromosome. For $A 8>G F P /+$ controls, males were also selected. All statistical analyses were performed using Prism 8 software (GraphPad). Average and SEM are reported. Outliers are determined by Q-test and excluded from the sample pools. For each data point, at least eight animals per genotype were dissected and at least two biological replicates were examined. All data were assumed to follow a Gaussian distribution. As we were making comparisons within specific target cells, and not between targets, most comparisons were performed by Student's $t$ test (Welch's correction was used in cases of unequal variance) or Kolmogorov-Smirnov (K-S) test. Comparisons between target cells was performed with one-way ANOVA followed by Tukey's test.

\section{Results}

$1 \mathrm{~b}$ and $1 \mathrm{~s}$ inputs contribute to postsynaptic activity in a target-specific manner

Converging inputs contribute to the overall postsynaptic response. In this study, we examined to what extent one input can influence the structure and function of a convergent input. To address this, we first determined the activity contribution of each $\mathrm{MN}$ on the postsynaptic muscle target in a wild-type condition. We chose $\mathrm{m} 6, \mathrm{~m} 12$, and $\mathrm{m} 4$ because (1) prior studies showed that each $1 \mathrm{~b}$ contributes a unique percentage of the total postsynaptic activity, (2) these muscles have been frequently analyzed in NMJ studies (Nose, 2012; Menon et al., 2013), and (3) the $1 \mathrm{~b}$ and $1 \mathrm{~s}$ innervation patterns on these muscles enabled the identification of common and muscle-specific principles (all muscles have unique $1 \mathrm{~b}$ MNs, but $\mathrm{m} 6$ and $\mathrm{m} 12$ are innervated by the same $1 \mathrm{~s} \mathrm{MN).}$

We measured the $1 \mathrm{~b} \mathrm{MN}$ contribution to the total EPSP on a muscle by muscle basis. $1 \mathrm{~b}$ and $1 \mathrm{~s}$ axons fasciculate into nerve bundles as they exit the VNC, which impeded us from physically stimulating each neuron independently without patching each cell body (Choi et al., 2004). To circumvent this, we combined NMJ electrophysiology with a postsynaptically targeted genetically encoded calcium indicator, SynapGCaMP6f (MHC-CD8:: GCaMP6f-Sh; Newman et al., 2017). Some of the earliest 
evidence for the existence of two distinct MN populations was uncovered $>40$ years ago (Jan and Jan, 1976) through observations that different voltage injections elicited two populations of muscle EPSPs. It was later found that lower voltage injection generated action potentials in $1 \mathrm{~b}$ MNs (Lnenicka and Keshishian, 2000); therefore, we varied the stimulus protocol to independently elicit and record EPSPs from $1 \mathrm{~b}$ alone and $1 \mathrm{~b}+1 \mathrm{~s}$ together (see Materials and Methods). SynapGCaMP6f fluorescence changes at $1 \mathrm{~b}$ and $1 \mathrm{~s}$ NMJs confirmed whether the recorded EPSPs were because of $1 \mathrm{~b}$ alone or $1 \mathrm{~b}+1 \mathrm{~s}$ activity (Fig. $1 B$, Movie 1 ). Using this procedure, the average total EPSP amplitude $(1 \mathrm{~b}+1 \mathrm{~s})$ in $\mathrm{m} 6$ was $31.55 \mathrm{mV}$ and the average $1 \mathrm{~b}-$ derived EPSP was $17.17 \mathrm{mV}$ (Fig. 1C,F). Thus, the m6-1b MN contributes $54 \%$ of the total m6 EPSP (Fig. 1I). Interestingly, at $\mathrm{m} 12$ the $1 \mathrm{~b} \mathrm{MN}$ contributes $31 \%$ of the total EPSP, and at $\mathrm{m} 4$ the $1 \mathrm{~b} \mathrm{MN}$ represents $62 \%$ (Fig. $1 D, E, G-I$ ). Thus, we determined the contribution of each $\mathrm{MN}$ ( $1 \mathrm{~b}$ and $1 \mathrm{~s}$ ) to the postsynaptic muscle activity in wild-type larvae and found that the relative strength of each MN differed between muscles, with the m4-1b MN contributing the most and the m12-1b MN the least. Notably, m6-1b MN and m12-1b MN contributions are different although they are innervated by the same 1s MN. These data established a model in which to introduce perturbations and examine synaptic plasticity.

\section{Cell-specific genetic ablation of 1s MNs by ectopic hid,rpr expression}

To begin to examine whether one input can respond to perturbations in an adjacent input, we needed to disrupt one $\mathrm{MN}$ and monitor the impact on the convergent MN. Additionally, being able to disrupt one input before or after innervation can shed light on whether initial coinnervation is required for synaptic plasticity. We needed to identify drivers that are expressed specifically within subsets of the convergent neurons. In a previous study, we showed that $D I P-\alpha$ is expressed in subsets of neurons in the larval VNC, including interneurons and two 1s MNs, and that the removal of $D I P-\alpha$ impeded 1s innervation of $\mathrm{m} 4$ (Ashley et al., 2019). To gain genetic access specifically to the $1 \mathrm{~s}$ MNs, we examined a GAL4 driver derived from the DIP- $\alpha$ promoter. In the adult neuromuscular circuit, this driver [hereafter referred to as A8-GAL4 or (A8)] was found to be expressed in a small subset of MNs (Venkatasubramanian et al., 2019). We used A8-GAL4 to drive UAS-GFP and found expression in only two pairs of segmentally repeated neurons in the third instar VNC (Fig. 2A,B, arrows). The labeled neurons located ventrally in the VNC have axons that project medially and dorsally toward the neuropil (Fig. 2C, arrow). The other A8-expressing neurons are located in the dorsal region of the VNC and showed an ipsilateral projection with a large dendritic arbor (Fig. 2D, arrowhead). Examination of nerves exiting the VNC showed
A

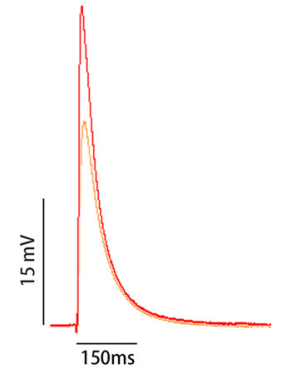

B
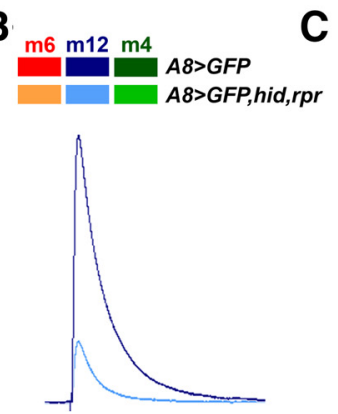

D

E
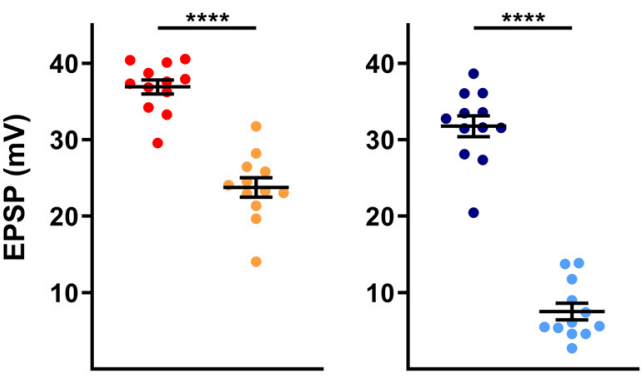

G

H
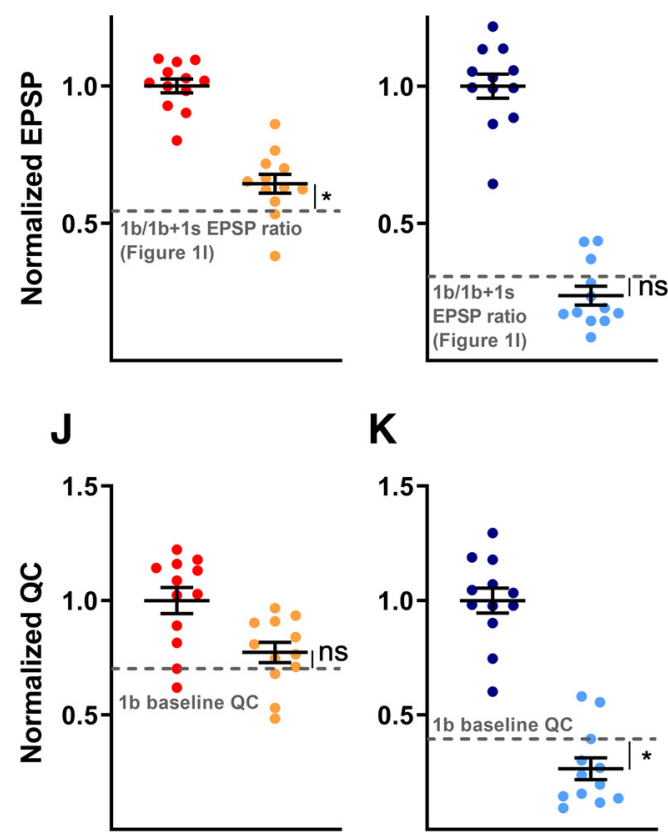

K

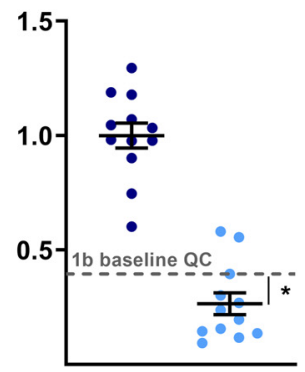

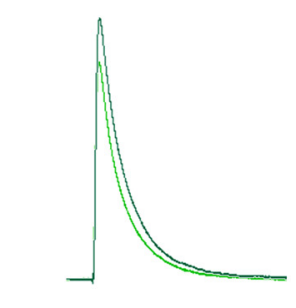

F

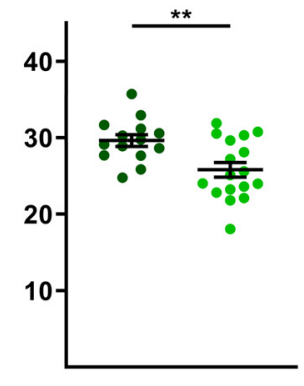

I

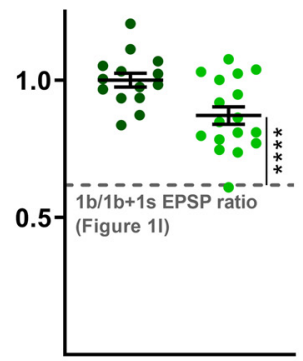

L

C

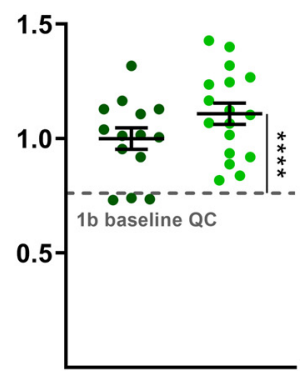

Figure 6. 1b NMJs elevate evoked neurotransmission in a target-specific manner in the absence of $1 \mathrm{~s}$ inputs. $\boldsymbol{A}-\boldsymbol{C}$, Representative EPSP traces of $\mathrm{m} 6(\boldsymbol{A}), \mathrm{m} 12(\boldsymbol{B})$, and $\mathrm{m} 4(\boldsymbol{C})$ in control (A8>GFP) and 1s-ablated (A8>GFP, hid,rpr) animals. Traces and graphs are color coded as indicated in the color key. $\boldsymbol{D}-\boldsymbol{F}$, Quantification of EPSP amplitudes in $\mathrm{m} 6\left(\boldsymbol{D} ; t_{(22)}=8.306, p<0.0001\right.$, unpaired $t$ test), $\mathrm{m} 12\left(\boldsymbol{E} ; t_{(22)}=13.82, p<0.0001\right.$, unpaired $t$ test), and $\mathrm{m} 4\left(\boldsymbol{F} ; t_{(29)}=3.057, p=0.0048\right.$, unpaired $t$ test $)$ in control and $1 \mathrm{~s}$-ablated animals. $\mathbf{G}-\mathbf{I}$, Normalized EPSPs of $\mathrm{m} 6\left(\boldsymbol{G} ; t_{(25)}=2.301, p=0.0300\right.$, unpaired $t$ test), $\mathrm{m} 12\left(\boldsymbol{H} ; t_{(25)}=1.552, p=0.1332\right.$, unpaired $t$ test), and $\mathrm{m} 4\left(\boldsymbol{I} ; t_{(31)}=4.605, p<0.0001\right.$, unpaired $t$ test $)$ in control and $1 \mathrm{~s}$-ablated third instar larvae. Normalized EPSPs in A8>GFP,hid,rpr are compared with the EPSP ratio of $1 \mathrm{~b} / 1 \mathrm{~b}+1 \mathrm{~s}$ calculated from corresponding muscles in Figure 11 , indicated by gray dashed line. Note m6-1b slightly compensates, m12-1b does not compensate and $\mathrm{m} 4$ largely compensates the loss of $1 \mathrm{~s}$ MNs. $\boldsymbol{J}-\boldsymbol{L}$, Normalized $Q \mathrm{C}$ of $\mathrm{m} 6\left(\boldsymbol{J} ; t_{(25)}=1.239, p=0.2268\right.$, unpaired $t$ test), $\mathrm{m} 12\left(\boldsymbol{K} ; t_{(25)}=2.119, p=0.0442\right.$, unpaired $t$ test), and $\mathrm{m} 4\left(\boldsymbol{L} ; t_{(31)}=4.639, p<0.0001\right.$, unpaired $t$ test) in control and 1s-ablated larvae. Normalized QCs were compared with $1 \mathrm{~b}$ baseline QC (see Materials and Methods). Note m4-1b shows an increased QC, while m12-1b shows a decrease. Error bars indicate \pm SEM. $* p<0.05$, $* * p<0.01$, ****p $<0.0001$, ns - not significant. $n$ values (NMJs/larva) are 12/9, 12/11, 12/9, 12/12, 14/11, and $17 / 14$, respectively. 
two axons in each bundle (Fig. $2 B, D$ asterisks), suggesting that these $A 8$-positive neurons are MNs. The dorsal neurons were colabeled with the transcription factor Eve, which labels three medial neurons, aCC, pCC, and MNISN-1s (Doe et al., 1988; Broadus et al., 1995), and thus these neurons were confirmed as MNISN-1s (also called the dCE) based on their location (Fig. 2B). Drosophila larval MNs make connections with their muscle targets with very high fidelity, allowing us to unequivocally determine the identity of the MNs based on their innervation pattern. In $A 8>G F P$ third instar larvae, one axon innervates the ventral muscles including muscles $6,7,12$, and 13 , similar to the connectivity pattern of $\mathrm{MNISNb} / \mathrm{d}-1 \mathrm{~s}$ (also called the vCE). The other axon innervates the dorsal muscles, corresponding to dCE (Figs. $1 A, 2 E, F$ ). To distinguish $1 \mathrm{~b}$ and 1s NMJs, we also stained for DLG as $1 \mathrm{~b}$ NMJs are surrounded by significantly more DLG (Guan et al., 1996; Fig. 2E,F). A8 is not expressed in the third $1 \mathrm{~s} \mathrm{MN}$ that innervates lateral transverse muscles. Importantly, $A 8$ does not label any other MNs but only a few additional cells in one segment of the VNC (Fig. $2 A$, carets). In summary, $A 8$ labels two $1 \mathrm{~s} \mathrm{MNs}$ (the vCE and $\mathrm{dCE}$ ) within each hemisegment.

We used A8-GAL4 to drive head involution defective (hid) and reaper (rpr) in $\mathrm{vCE}$ and $\mathrm{dCE}$. hid and rpr have important functions in programmed cell death, and ectopic expression of both genes more reliably induces neuronal death than either gene alone (Zhou et al., 1997; Pauls et al., 2015). As shown in Figure $2 A-F, A 8>G F P$ robustly labels $1 \mathrm{~s} \mathrm{MNs}$ but in third instar larvae that ectopically express hid, $r p r(A 8>G F P, h i d, r p r)$, all vCE or dCE cell bodies and NMJs are absent (Fig. 2G-J). Thus, the expression of hid, rpr is sufficient to genetically ablate 1s MNs.

To determine whether cell death occurred before or after innervation, we examined earlier developmental stages and visualized GFP in $1 \mathrm{~s}$ MN cell bodies and NMJs in $A 8>G F P$ and $A 8>G F P$,hid,rpr. Neuromuscular innervation is established at embryonic stage 16 (Halpern et al., 1991; Broadie and Bate, 1993; Yoshihara et al., 1997), so we focused on stage 15 and later stages including stage 17 and first instar larvae. In stage 15 embryo controls $(A 8>G F P)$, only a subset of 1 s MNs are detected since not all dCE cell bodies (Eve positive) are costained with GFP and no vCE cell bodies are observed (Fig. $3 A, B$ ). Agematched $A 8>G F P$, hid, $r p r$ embryos showed GFP expression patterns similar to those of controls (Fig. 3C,D); thus, no cell death occurred before neuromuscular innervation. By embryonic stage 17, more vCE and dCE cell bodies expressed $A 8$ but the lack of GFP in some cells suggests that $A 8$ expression has not reached maximal levels (Fig. $3 E, F$ ). In stage $17 A 8>G F P$, hid, rpr embryos, cells were undergoing apoptosis, as revealed by significant decreases in GFP and by the loss of Eve staining (suggesting nuclear degradation; Fig. $3 G, H$ ). Finally, in $A 8>$ GFP early first instar larvae, all vCE and dCE cell bodies were labeled (Fig. 3I,J) and NMJs were present (Fig. 3M). Age-matched A8> GFP, hid, rpr larvae completely lacked vCE and $\mathrm{dCE}$ cell bodies (Fig. $3 K, L$ ), although some 1s NMJs were still observed (Fig. 3M,N), suggesting that $1 \mathrm{~s}$ NMJs were established before ablation in this genetic background. By late first instar stage, $A 8>$ GFP, hid, rpr larvae show no traces of $1 \mathrm{~s}$ NMJs on $\mathrm{m} 6, \mathrm{~m} 12$, and $\mathrm{m} 4$ (Fig. 3O). Together, A8-GAL4 is specifically expressed in vCE and dCE, and driving ectopic expression of cell death genes with $A 8$ triggers apoptosis in 1s MNs after synaptic contacts are established.

$1 \mathrm{~b}$ NMJs expand upon loss of adjacent $1 \mathrm{~s}$ MNs

NMJ structural plasticity can be induced by the perturbation of synaptic function (Budnik et al., 1990; Jarecki and Keshishian,
A

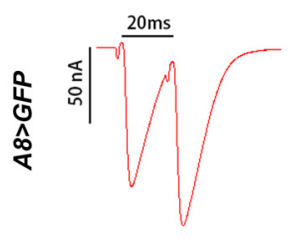

B
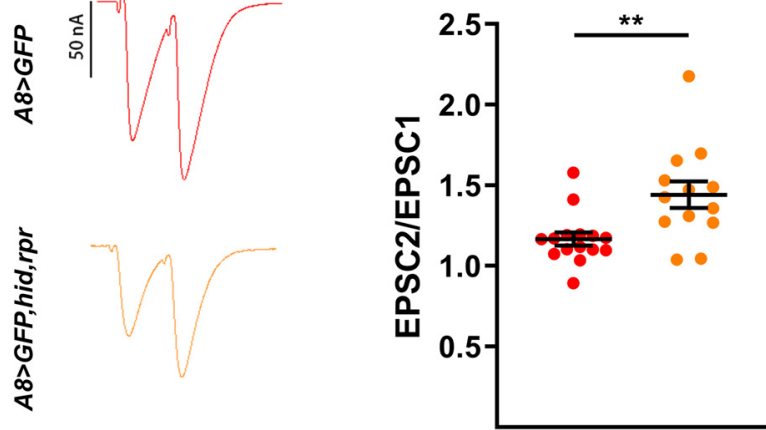

Figure 7. Overall paired-pulse ratio is increased upon ablation of 15 MNs. $\boldsymbol{A}$, Representative paired-pulse recordings of $\mathrm{m} 6$ in control $(A 8>G F P)$ and 1s-ablated (A8> GFP,hid,rpr) animals. B, Quantification of paired-pulse ratio (EPSC2/EPSC1) in control (red) and 1s-ablated animals (orange; $t_{(26)}=3.120, p=0.0044$, unpaired $t$ test). $n$ values (NMJs/larva) are 15/10,13/10, respectively. Error bars indicate \pm SEM. $* * p<0.01$.

1995; Sigrist et al., 2003; Goel et al., 2019a; Perry et al., 2020). Here, we examined whether the loss of $1 \mathrm{~s} \mathrm{MNs} \mathrm{can} \mathrm{induce} \mathrm{struc-}$ tural changes at adjacent 1b NMJs. The size of each NMJ is well characterized (represented by the number of boutons), and this allows us to observe structural changes because of perturbations. We genetically ablated $1 \mathrm{~s}$ MNs (vCE and dCE) and counted the number of boutons on $\mathrm{m} 6, \mathrm{~m} 12$, and $\mathrm{m} 4$ in wandering third instar larvae (NMJ expansion is complete; Li et al., 2002). We observed an increase in the number of $1 \mathrm{~b}$ boutons on all three muscles (Fig. $4 A-G$ ) when comparing them to $A 8>G F P$ controls, suggesting that $1 \mathrm{~b}$ NMJs expanded when adjacent $1 \mathrm{~s}$ MNs were ablated. Because m4-1s innervation frequency is $\sim 80 \%$ in wild type (Ashley et al., 2019; Aponte-Santiago et al., 2020), we used $A 8>G F P$ to confirm the presence of $\mathrm{m} 4-1 \mathrm{~s}$ in controls and excluded muscles lacking 1 s innervation. Interestingly, we also found an increase in small budding boutons, called satellite boutons, emanating from $1 \mathrm{~b}$ boutons (Fig. $4 D-F$, insets, $H$ ). These structures represent immature but functional boutons as they contain all synaptic machinery and postsynaptic receptors (Torroja et al., 1999; Dickman et al., 2006; O'Connor-Giles et al., 2008; O'Connor-Giles and Ganetzky, 2008; Lee and Wu, 2010; Carrillo et al., 2015). Thus, all 1b NMJs initiate structural plasticity mechanisms to respond to the loss of adjacent $1 \mathrm{~s}$ MNs.

\section{$1 \mathrm{~b}$ NMJs elevate their rate of spontaneous release upon loss of 1 s MNs}

To determine to what extent a synaptic input can influence the functional synaptic plasticity of a converging input, we first examined $1 \mathrm{~b}$ spontaneous neurotransmitter release at muscles where $1 \mathrm{~s}$ MNs were ablated after innervation. $1 \mathrm{~b}$ and $1 \mathrm{~s} \mathrm{MNs}$ have unique spontaneous release properties. For example, 1bderived spontaneous events (stimulus-independent release of neurotransmitter vesicles; also referred to as mEPSPs) have smaller amplitudes compared with 1s-derived mEPSPs (Nguyen and Stewart, 2016; Newman et al., 2017). Therefore, the ablation of $1 \mathrm{~s}$ inputs should shift the mean mEPSP amplitude toward the smaller 1b-like amplitude if there is no compensation. We performed current-clamp recordings from $\mathrm{m} 6, \mathrm{~m} 12$, and $\mathrm{m} 4$. Indeed, $A 8>G F P$, hid, $r p r$ revealed decreased mEPSP amplitudes compared with $A 8>G F P$ controls, and a significant shift in cumulative amplitude probability distribution (Fig. 5A-G). Because of the inability of standard NMJ electrophysiology experiments to distinguish between $1 \mathrm{~b}$ and $1 \mathrm{~s}$ mEPSPs in controls, we are unable to unambiguously determine whether the $1 \mathrm{~b}$ mEPSP 

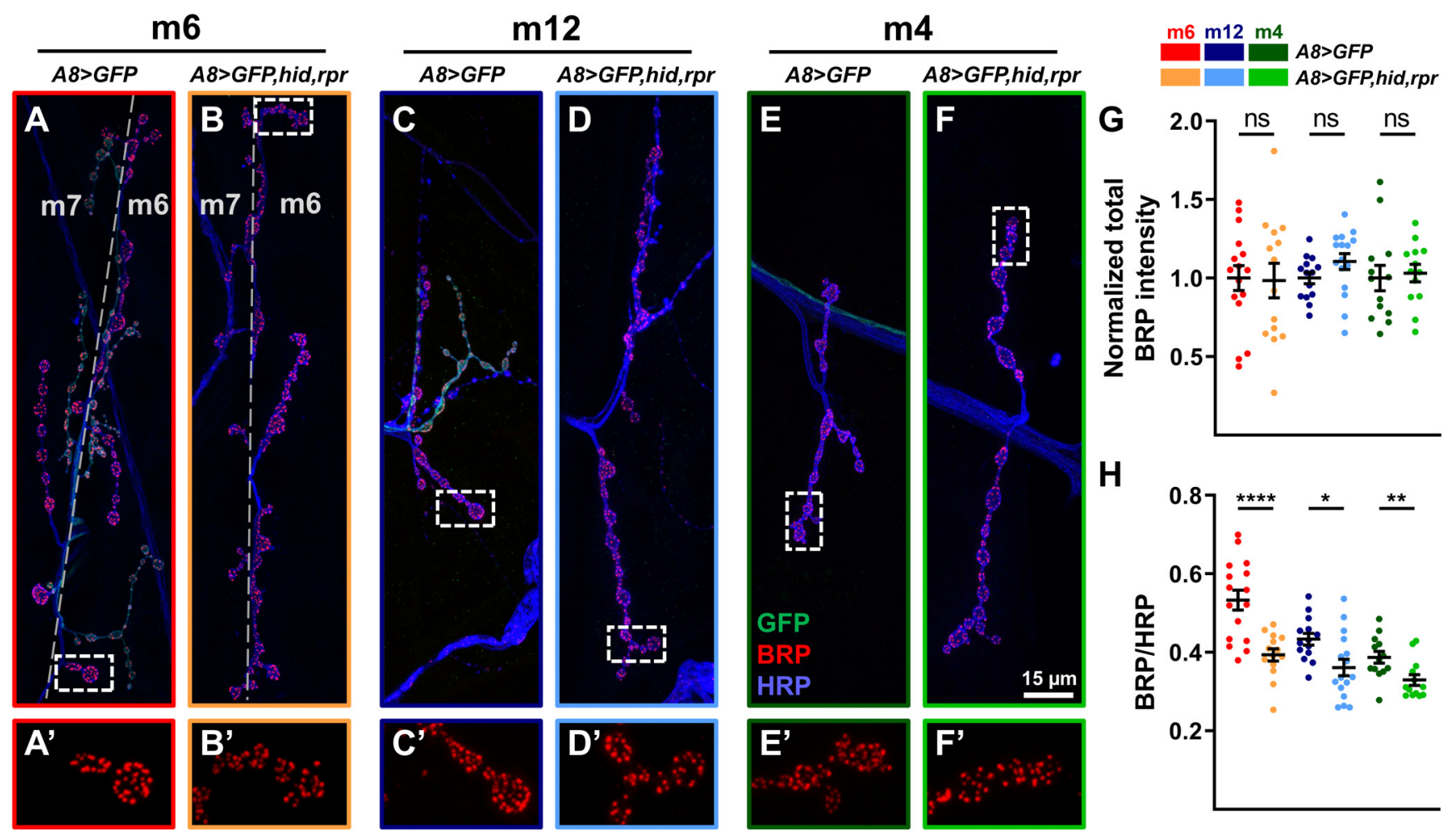

H

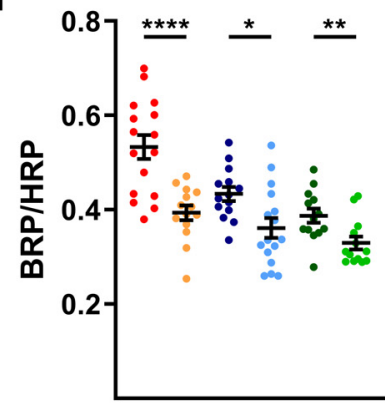

Figure 8. Quantification of BRP levels at 1b NMJs. $\boldsymbol{A}-\boldsymbol{F}$, Representative BRP immunostaining from $\mathrm{m} 6(\boldsymbol{A}, \boldsymbol{B}), \mathrm{m} 12(\boldsymbol{C}, \boldsymbol{D})$, and $\mathrm{m} 4(\boldsymbol{E}, \boldsymbol{F})$ in control (A8>GFP) and 1s-ablated (A8>GFP, hid, $r p r$ ) animals labeled with GFP (green), BRP (red), and HRP (blue). $\boldsymbol{A}^{\prime}-\boldsymbol{F}^{\prime}, 2.5 \times$ zoomed 1b boutons corresponding to dashed regions in $\boldsymbol{A}-\boldsymbol{F}$ showing BRP staining. $\mathbf{G}$, Quantification of normalized total BRP intensity of 1b NMJs on $\mathrm{m} 6\left(t_{(28)}=0.1199, p=0.9054\right.$, unpaired $t$ test), $\mathrm{m} 12$ ( $t_{(28)}=1.627, p=0.1148$, unpaired $t$ test), and $\mathrm{m} 4\left(t_{(24)}=0.3189, p=0.7525\right.$, unpaired $t$ test). Images and graphs are color coded as indicated in the color key. $\boldsymbol{H}$, Quantification of BRP/HRP sum intensity ratio (density) from $\mathrm{m} 6\left(t_{(24.31)}=4.705, p<0.0001\right.$, unpaired $t$ test with Welch's correction), $\mathrm{m} 12\left(t_{(28)}=2.736, p=0.0107\right.$, unpaired $t$ test), and $\mathrm{m} 4\left(t_{(24)}=2.847, p=0.0089\right.$, unpaired $t$ test). Error bars indicate $\pm \mathrm{SEM} . * p<0.05, * * p<0.01, * * * * p<0.0001, \mathrm{~ns}$ not significant. $n$ values (NMJs/larva) are 16/8, 14/8, 14/8, 16/8, 13/7, and 13/7, respectively.

amplitudes are affected by the loss of 1s inputs. Nonetheless, we can conclude that $1 \mathrm{~b}$ NMJs cannot fully restore the average mEPSP amplitudes to wild-type levels.

Another measure of stimulus-independent activity is the rate of spontaneous neurotransmitter release. In prior studies, mEPSP frequencies were found to be higher at $1 \mathrm{~b}$ NMJs than $1 \mathrm{~s}$ NMJs (e.g., $2.3 \mathrm{~Hz}$ at m4-1b NMJ and $1 \mathrm{~Hz}$ at m4-1s NMJ; Newman et al., 2017); thus, if the elimination of 1s MNs does not affect the rate of $1 \mathrm{~b}$ spontaneous release, overall mEPSP frequencies should decrease by about one-third. However, we did not observe any reduction of $\mathrm{mEPSP}$ frequencies on $\mathrm{m} 6, \mathrm{~m} 12$, and $\mathrm{m} 4$ when comparing $A 8>G F P$ and $A 8>G F P$, hid,rpr animals, and $\mathrm{m} 12$ even showed an increased rate of spontaneous release (Fig. 5A-C,H). Importantly, these spontaneous events correspond to $1 \mathrm{~b}$-derived mEPSPs since (1) the mean amplitudes decrease (Fig. 5G), and (2) there are no remaining 1s NMJs (Fig. $2 I, J)$. These results strongly suggest that $1 \mathrm{~b} \mathrm{MN}$ synaptic plasticity mechanisms detect the loss of convergent 1 s MNs and elevate their rate of spontaneous neurotransmitter release. Spontaneous neurotransmitter release has been implicated in synaptic maturation (Joseph et al., 2012; Choi et al., 2014; Andreae and Burrone, 2015, 2018; Cho et al., 2015; Kavalali, 2015), and, thus, the increased mEPSP frequencies we observe could underlie the increased $1 \mathrm{~b}$ bouton numbers.

1b MNs elevate evoked neurotransmission upon loss of $1 \mathrm{~s}$ MNs in a target-specific manner

Having demonstrated $1 \mathrm{~b}$ synaptic plasticity of spontaneous activity, we next examined whether $1 \mathrm{~b}$-evoked neurotransmission could also be modified by the loss of 1 s MNs. Unlike spontaneous neurotransmitter release, EPSPs require stimulation to depolarize the presynaptic neuron above threshold. This suprathreshold stimulation triggers an action potential to induce neurotransmitter release and elicit a postsynaptic response. To examine whether $1 \mathrm{~b}$ MNs can compensate for the loss of 1s synaptic drive, we recorded EPSPs in both $A 8>G F P$ and $A 8>G F P$, hid,rpr (Fig. 6A-F). To determine whether 1b-derived EPSP amplitudes are affected by the loss of 1 s inputs, we normalized the EPSPs (A8>GFP, hid, rpr EPSP/A8>GFP EPSP) and compared these values to the calculated $1 \mathrm{~b} / 1 \mathrm{~b}+1 \mathrm{~s}$ ratio in Figure $1 I$. This analysis allows for the comparison of $1 \mathrm{~b}$-derived EPSPs with and without convergent $1 \mathrm{~s}$ MNs.

We observed target-specific changes in 1b-derived EPSPs (Fig. 6G-I). At $\mathrm{m} 4$, we observed a significant increase in 1bderived EPSPs compared with the control $1 b / 1 b+1 s$ ratio (Fig. $6 I$ ). By repeating the analysis at other muscles, we found a mild increase in 1b-derived EPSPs at m6 (Fig. 6G) and, surprisingly, no change at $\mathrm{m} 12$ (Fig. $6 H$ ). When comparing the degree of EPSP compensations (Fig. $6 G-I$ ) to the wild-type 1 b contribution to total EPSP at each muscle (Fig. 1I), a pattern arises whereby the m4-1b has both the most compensation and the largest $1 \mathrm{~b}$ contribution. Meanwhile, $\mathrm{m} 12-1 \mathrm{~b}$ did not compensate and displayed the smallest $1 \mathrm{~b}$ contribution.

Next, we examined QC, a calculation of the approximate amount of neurotransmitter released per stimulation. We found that the normalized QC of m4-1b in A8> GFP, hid,rpr was significantly increased compared with the m4-1b baseline QC (see Materials and Methods), whereas there was no change at m6-1b, and even a decrease at m12-1b (Fig. 6J-L). To test whether the loss of 1s MNs perturbed short-term 1b NMJ plasticity, we 

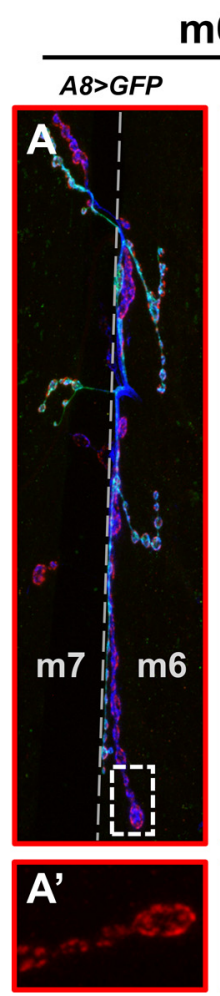

m6
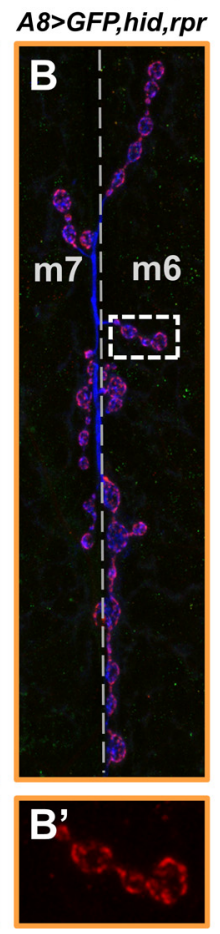
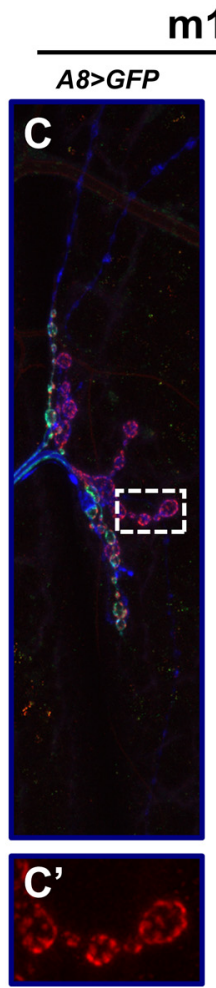

m12
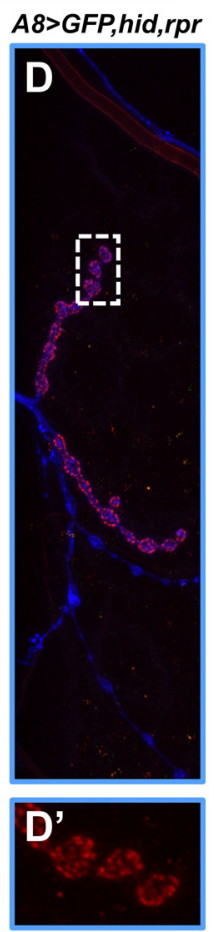
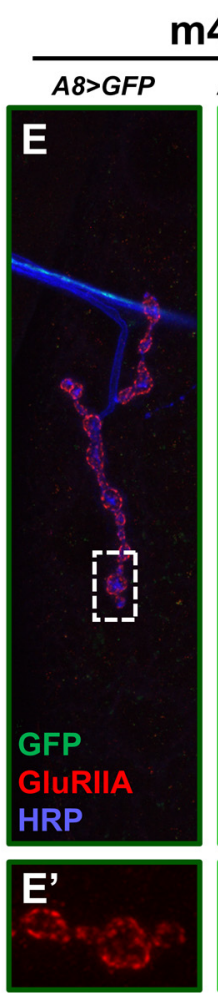

m4
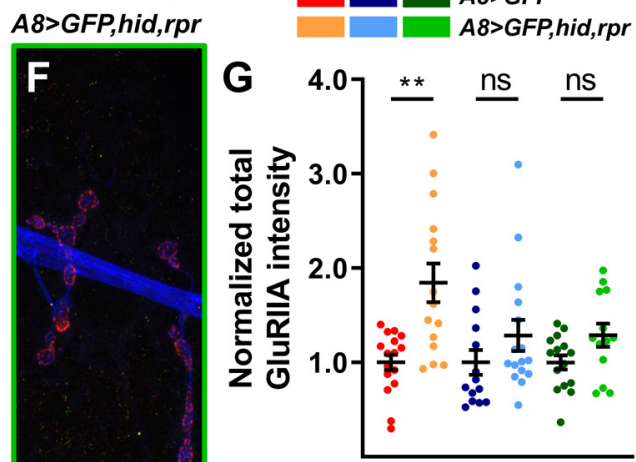

H

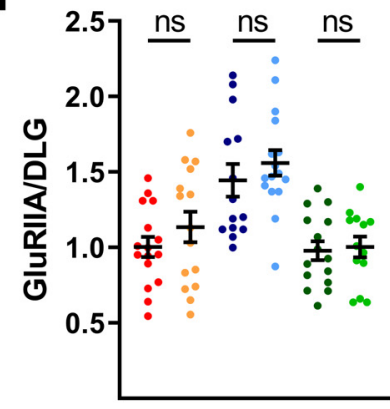

Figure 9. Quantification of GluRlIA levels at 1b NMJs. $\boldsymbol{A}-\boldsymbol{F}$, Representative GluRllA immunostaining from m6 $(\boldsymbol{A}, \boldsymbol{B}), \mathrm{m} 12(\boldsymbol{C}, \boldsymbol{D})$, and $\mathrm{m} 4(\boldsymbol{E}, \boldsymbol{F})$ in control $(A 8>$ GFP) and 1s-ablated animals (A8>GFP,hid,rpr) labeled with GFP (green), GluRIIIA (red), and DLG (blue). $\boldsymbol{A}^{\prime}-\boldsymbol{F}^{\prime}, 2.5 \times$ zoomed 1b boutons corresponding to dashed regions in $\boldsymbol{A}-\boldsymbol{F}$ showing GluRllA staining. $\boldsymbol{G}$, Quantification of normalized total GluRIIA intensity of $\mathrm{m} 6\left(t_{(18.38)}=3.812, p=0.0012\right.$, unpaired $t$ test with Welch's correction), $\mathrm{m} 12$ ( $t_{(28)}=1.337, p=0.1921$, unpaired $t$ test), and $\mathrm{m} 4$ ( $t_{(26)}=$ 2.049, $p=0.0507$, unpaired $t$ test). Images and graphs are color coded as indicated in the color key. $\boldsymbol{H}$, Quantification of GluRIIA/DLG sum intensity ratio (density) of m6 $\left(t_{(29)}=1.108\right.$, $p=0.2771$, unpaired $t$ test), $\mathrm{m} 12\left(t_{(28)}=0.8544, p=0.4002\right.$, unpaired $t$ test), and $\mathrm{m} 4\left(t_{(26)}=0.2679, p=0.7909\right.$, unpaired $t$ test). Error bars indicate \pm SEM. $* * p<0.01$, ns - not significant. $n$ values (NMJs/larva) are 16/8, 15/8, 14/7, 16/8, 15/8, and 13/7, respectively.

examined paired-pulse facilitation $(\mathrm{PPF})$ as it is a measure of evoked release probability $\left(P_{r}\right)$. We measured $\mathrm{m} 6 \mathrm{PPF}$ in $A 8>G F P$, hid, rpr and found an enhancement compared with $A 8>$ GFP controls (Fig. 7). Although these data suggest an increase in $1 \mathrm{~b}$ paired-pulse ratio (PPR), this can also be explained by the loss of 1s-specific synaptic depression (Newman et al., 2017) as both MNs are stimulated in controls. Furthermore, we were unable to definitively isolate $1 \mathrm{~b}$-specific PPF in controls as the second EPSC could be either $1 \mathrm{~b}$ derived or $1 \mathrm{~b}+1 \mathrm{~s}$ derived.

Overall, when ablating 1s MNs after NMJ formation, 1b MNs that innervate the same muscle upregulated their rate of spontaneous release, and, importantly, the m4-1b MN significantly compensated the total EPSP by increasing neurotransmitter release.

Active zone and glutamate receptor levels are unaltered by ablation of $1 \mathrm{~s}$ MNs

One way to increase both spontaneous and evoked release is by the addition of more AZs. We stained for BRP, an AZ scaffolding protein (Kittel et al., 2006), to visualize 1b AZs in the presence or absence of convergent $1 \mathrm{~s}$ MNs. Despite elevated $1 \mathrm{~b}$ synaptic drive, we observed no change in overall $1 \mathrm{~b}$ AZ intensity (Fig. $8 A-G$ ), although the larger NMJ sizes (Fig. 4G) led to reduced AZ densities (BRP/HRP; Fig. $8 H$ ). These data suggest that the number of AZs remains constant although NMJ size increases, similar to previous studies (Goel et al., 2019a, b; AponteSantiago et al., 2020; Goel et al., 2020).

Changes in the postsynaptic glutamate receptor density or localization can contribute to functional responses. To test whether the functional synaptic plasticity we observed was because of changes in glutamate receptor levels, we visualized the GluRIIA (Petersen et al., 1997). m6-1b NMJs showed a significant increase in GluRIIA intensity, whereas m12-1b and m4-1b did not show a significant increase (Fig. 9A-G). Interestingly, $1 \mathrm{~b}$ NMJ GluRIIA density (Fig. 9H) was unaffected, suggesting that receptor abundance may not contribute to $1 \mathrm{~b}$ synaptic plasticity.

Overall, these data indicate that presynaptic BRP or postsynaptic GluRIIA levels are not an integral component of $1 \mathrm{~b}$ synaptic plasticity induced by the ablation of $1 \mathrm{~s}$ MNs.

\section{Loss of the 1s-derived EPSP triggers $1 \mathrm{~b}$ synaptic plasticity}

Ablation is a crude perturbation that completely removes all $1 \mathrm{~s}$ MN synaptic activity and signaling pathways. Here, we focus on 1s evoked neurotransmission to examine whether its removal mimics the ablation of $1 \mathrm{~s}$ MNs. Tetanus toxin light chain (TNT) is a clostridial toxin that cleaves the vesicle SNARE synaptobrevin, which is required for calcium-dependent vesicle release (Sweeney et al., 1995). Ectopic expression of TNT blocks evoked neurotransmission without affecting spontaneous release and synaptic morphology (Sweeney et al., 1995; Aponte-Santiago et al., 2020). Therefore, we expressed TNT with A8-GAL4 to specifically block 1s-derived EPSPs and measured $1 \mathrm{~b}$ responses. We focused on m4-1b NMJs as these showed robust EPSP compensation, and we observed similar $1 \mathrm{~b}$ synaptic plasticity when compared with ablation of $1 \mathrm{~s}$ MNs. Structurally, the m4-1b NMJ was larger (Fig. 10A-C) and displayed more satellite boutons (Fig. $10 D)$. Functionally, we observed an increase in overall mEPSP frequency (Fig. 10E,F) and 1b-derived EPSP amplitude (when 

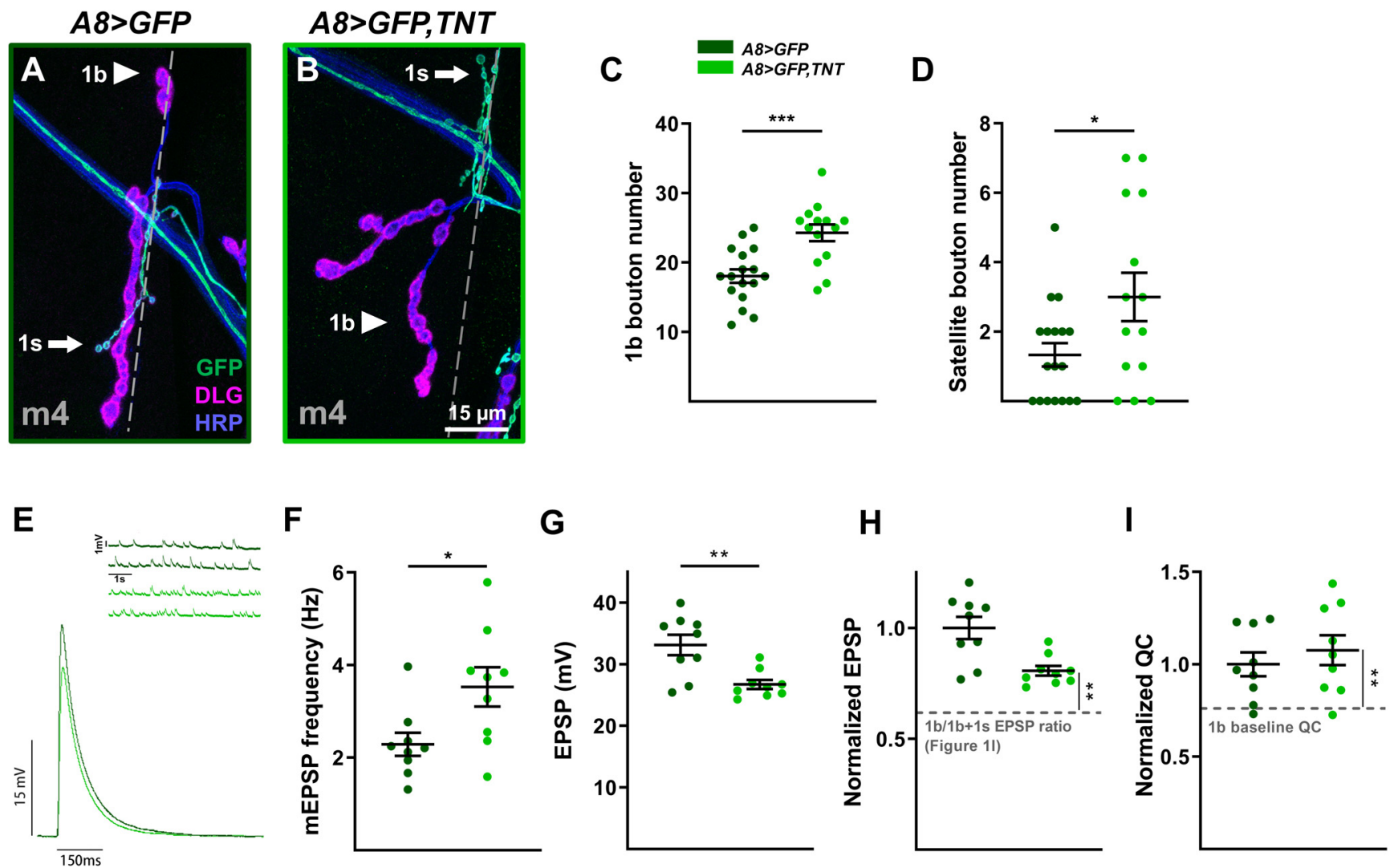

Figure 10. Loss of $1 \mathrm{~s}$ evoked neurotransmission contributes to $1 \mathrm{~b}$ synaptic plasticity. $\boldsymbol{A}, \boldsymbol{B}$, Representative m4 NMJs labeled with GFP (green), DLG (magenta), and HRP (blue) in control ( $\boldsymbol{A}$; A8> GFP: dark green) and 1s-silenced (B; A8>GFP,TNT: light green) animals. C, Quantification of $1 \mathrm{~b}$ boutons on $\mathrm{m} 4$ in control and 1s-silenced animals $\left(t_{(29)}=4.099, p=0.0003\right.$, unpaired $t$ test). Images, representative traces, and graphs are color coded as indicated in the color key. $\mathbf{D}$, Quantification of satellite boutons from $\mathrm{m} 4$ in control and $1 \mathrm{~s}$-silenced animals $\left(t_{(18.90)}=2.161\right.$, $p=0.0437$, unpaired $t$ test with Welch's correction). $\boldsymbol{E}$, Representative mEPSP and EPSP recordings in control and 1s-silenced animals. $\boldsymbol{F}$, Quantification of $\mathrm{mEPSP}$ frequencies from $\mathrm{m} 4$ in control and 1s-silenced animals ( $t_{(16)}=2.515, p=0.0230$, unpaired $t$ test). G, Quantification of EPSP amplitudes in control and 1s-silenced animals $\left(t_{(11.01)}=3.529, p=0.0047\right.$, unpaired $t$ test with Welch's correction). $\boldsymbol{H}$, Normalized EPSP from $\mathrm{m} 4$ in control and 1s-silenced animals $\left(t_{(20.75)}=3.736, p=0.0012\right.$, unpaired $t$ test with Welch's correction). Normalized EPSP of 1s-silenced animals is compared with the EPSP ratio of $1 \mathrm{~b} / 1 \mathrm{~b}+1 \mathrm{~s}$ calculated from $\mathrm{m} 4$ in Figure $1 /$, indicated by gray dashed line. $I$, Normalized $Q C$ from $\mathrm{m} 4$ in control and $1 \mathrm{~s}$-silenced animals $\left(t_{(23)}=\right.$ 3.159, $p=0.0044$, unpaired $t$ test). $Q C$ from 1s-silenced $\mathrm{m} 4$ was estimated using corrected EPSP and estimated 1b-derived mEPSP amplitude. Normalized QC was compared with $1 \mathrm{~b}$ baseline QC (see Materials and Methods). Error bars indicate \pm SEM. $* p<0.05, * * p<0.01, * * * p<0.001$. $n$ values (NMJs/larva) are 14/10, 17/9 for $\boldsymbol{C}$ and $\boldsymbol{D}$, and $9 / 7,9 / 6$ for $\boldsymbol{E}-\boldsymbol{I}$.

comparing the normalized EPSP to the $1 b / 1 b+1 s$ ratio; Fig. $10 G, H)$. The compensatory $1 \mathrm{~b}$-derived EPSP, however, was less than that observed with 1s ablation (Fig. 6I). QC in A8>GFP, TNT was calculated as before (see Materials and Methods; $1 \mathrm{~b}$ baseline QC), then normalized and compared with $1 \mathrm{~b}$ baseline QC. Importantly, we observed a similar increase in QC (Fig. 10I). These results suggest that the loss of 1s-derived EPSPs was sufficient to trigger $1 \mathrm{~b}$ synaptic plasticity, although other $1 \mathrm{~s}$ derived signals likely contribute since the ablation of 1 s induces a more robust $1 \mathrm{~b}$ response.

Robust $1 \mathrm{~b}$ synaptic plasticity requires initial $1 \mathrm{~s}$ innervation Above, we examined the structural and functional synaptic plasticity of one neuron when a converging neuron is ablated after innervation $(A 8>G F P$, hid,rpr). To probe whether initial coinnervation is required for plasticity, we explored another context when the 1s NMJ never forms on the postsynaptic target. In a prior study, we found that $D I P-\alpha$ is required for the recognition of $\mathrm{m} 4$ by the $\mathrm{dCE}$ (Fig. $11 A, B$ ), and we confirmed that no $1 \mathrm{~s}$ boutons are observed on $\mathrm{m} 4$ in $D I P-\alpha^{\text {null }}$ early first instar larvae (Fig. $11 C)$. Quantification of $\mathrm{m} 4-1 \mathrm{~b}$ boutons and satellite boutons in $D I P-\alpha^{\text {null }}$ mutants showed no difference from controls (Fig. 11D, $E)$. Also, in DIP- $\alpha^{\text {null }}$ mutants, the m 4 mEPSP frequency was significantly reduced (Fig. $11 F, G$ ), in contrast to the 1 s ablation (Fig. $5 H$ ). These results suggest that the m4-1b NMJ failed to compensate when the corresponding 1s NMJ never formed. Next, we examined EPSPs (Fig. 11F,H). Surprisingly, the lack of 1 s innervation in $D I P-\alpha^{\text {null }}$ mutant larvae revealed only a slight increase in the 1b-derived EPSP amplitude on $\mathrm{m} 4$ (Fig. 11I) and no change in QC (Fig. 11J). Thus, m4-1b synaptic plasticity is not as robust in $D I P-\alpha^{\text {null }}$ larvae, compared with $A 8>G F P$, hid,rpr.

Next, we examined $1 \mathrm{~b}$ structural and functional plasticity in another context where $\mathrm{m} 4$ is naturally missing $1 \mathrm{~s}$ innervation. In wild-type larvae, $\sim 20 \%$ of m4s lack 1s NMJs (Fig. 11C, control), which could underlie why $\mathrm{m} 4-1 \mathrm{~b} \mathrm{MNs}$ are able to compensate for 1s MN perturbation. However, comparing wild-type m4s that naturally lack $1 \mathrm{~s}$ innervation with those that have $1 \mathrm{~s}$ innervation, we observed no change in $1 \mathrm{~b}$ bouton numbers (Fig. 12A, $B)$. Similarly, 1b-derived mEPSP, EPSP and QC were unaffected (Fig. 12C-F). This confirms that lack of synaptic plasticity in $D I P-\alpha^{\text {null }}$ larvae is not because of the genetic manipulation. Further, these data show that m4-1b MNs that naturally lack adjacent 1s MNs are unable to compensate, suggesting that robust $\mathrm{m} 4-1 \mathrm{~b}$ synaptic plasticity is not because of the variable $1 \mathrm{~s}$ innervation observed on $\mathrm{m} 4$, but instead, induced when adjacent neurons are perturbed (for our model, see Discussion).

\section{Discussion}

The major gap that this article addresses is to what extent one synaptic input can influence the structural and functional 

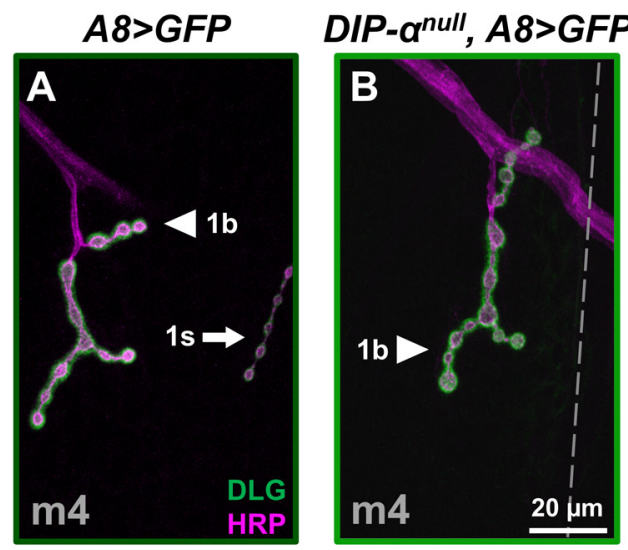

C

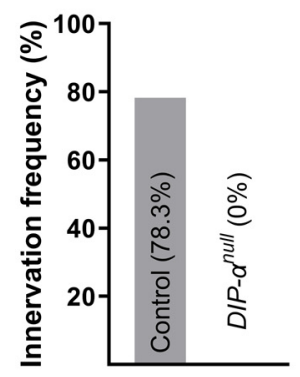

G

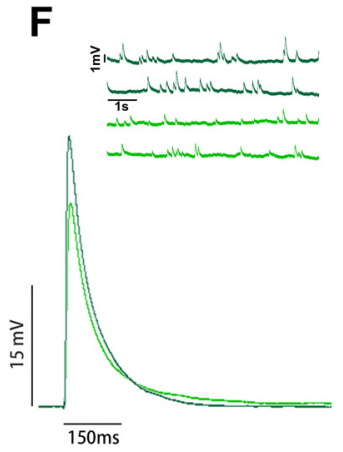

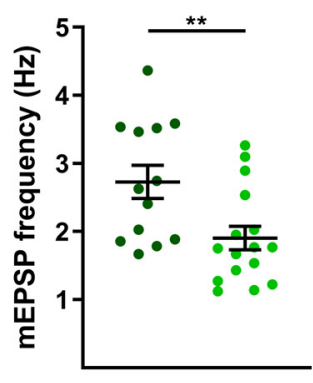

H

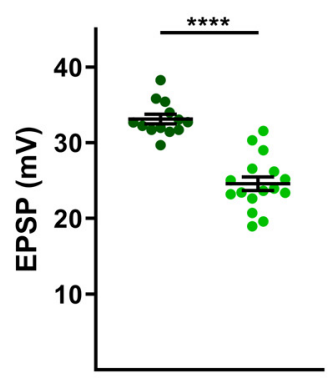

D

$A 8>G F P$

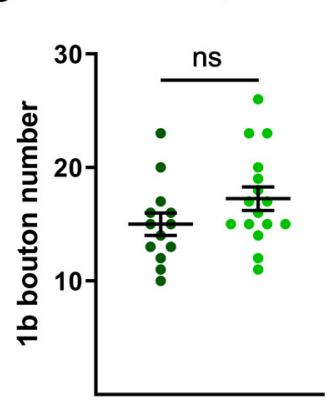

E

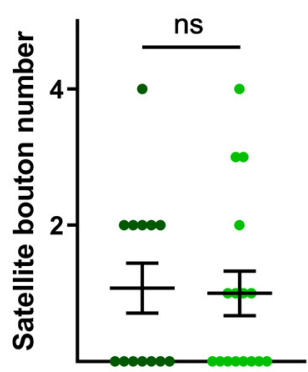

I

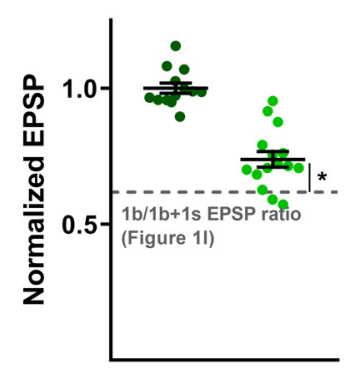

J

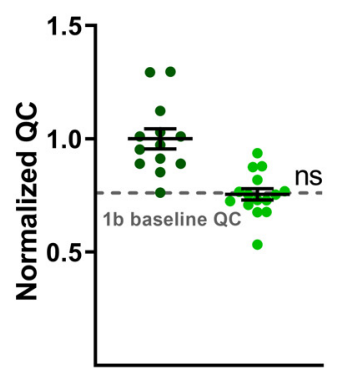

Figure 11. Robust $1 \mathrm{~b}$ MN synaptic plasticity requires initial 1s innervation. $\boldsymbol{A}, \boldsymbol{B}$, Representative $\mathrm{m} 4$ NMJs labeled with DLG (green) and HRP (magenta) in control (A8>GFP: dark green; $\boldsymbol{A}$ ) and mutant (DIP- $\alpha^{\text {null }}, A 8>$ GFP: light green; $\left.\boldsymbol{B}\right)$ animals. C, Quantification of $1 \mathrm{~s}$ innervation frequency in control and mutant first instar larvae. No m4-1s NMJs observed in mutants. $\boldsymbol{D}$, Quantification of $1 \mathrm{~b}$ boutons from $\mathrm{m} 4$ in control and mutant animals $\left(t_{(27)}=1.552, p=0.1323\right.$, unpaired $t$ test). Images, representative traces, and graphs are color coded as indicated in the color key. $\boldsymbol{E}$, Quantification of satellite boutons from $\mathrm{m} 4$ in control and mutant animals $\left(t_{(27)}=0.1563, p=0.8770\right.$, unpaired $t$ test). $\boldsymbol{F}$, Representative mEPSP and EPSP recordings from control and mutant animals. G, Quantification of mEPSP frequencies from $\mathrm{m} 4$ in control and mutant animals $\left(t_{(27)}=2.824, p=0.0088\right.$, unpaired $t$ test). $\boldsymbol{H}$, Quantification of EPSP amplitudes in control and mutant animals $\left(t_{(27)}=7.505, p<0.0001\right.$, unpaired $t$ test). $I$, Normalized EPSPs from $\mathrm{m} 4$ in control and mutant animals $\left(t_{(29)}=2.215, p=0.0348\right.$, unpaired $t$ test). Normalized mutant EPSP is compared with the EPSP ratio of $1 \mathrm{~b} / 1 \mathrm{~b}+1 \mathrm{~s}$ calculated from $\mathrm{m} 4$ in Figure 11 , indicated by gray dashed line. Note that $\mathrm{m} 4-1 \mathrm{~b}$-derived EPSP increases $23 \%$ in DIP- $\alpha$ mutants but increases $45 \%$ in $A 8>$ GFP, hid, $r p r$ (Fig. 6). J, Normalized QC from $\mathrm{m} 4$ in control and mutant animals $\left(t_{(20.14)}=0.086, p=0.9326\right.$, unpaired $t$ test with Welch's correction). Normalized QC was compared with $1 \mathrm{~b}$ baseline $Q C$ (see Materials and Methods). Error bars indicate \pm SEM. $* p<0.05, * * p<0.01, * * * * p<0.0001$, ns - not significant. $n$ values (NMJs/larva) are 46/8 and 20/4 for $\boldsymbol{B}$ and $\boldsymbol{C} ; 13 / 8$ and $16 / 8$ for $\boldsymbol{D}$ and $\boldsymbol{E}$; and $13 / 10$ and $16 / 10$ for $\boldsymbol{F}-\boldsymbol{J}$.

plasticity of a converging input. Here, we examine the Drosophila neuromuscular circuit and demonstrate $1 \mathrm{~b}$ synaptic plasticity induced by the loss of convergent $1 \mathrm{~s}$ MNs. The muscles examined in this study, $\mathrm{m} 6, \mathrm{~m} 12$, and $\mathrm{m} 4$, are coinnervated by unique $1 \mathrm{~b}-1 \mathrm{~s}$ MN pairs. First, we established an activity baseline in wild type and uncovered that $1 \mathrm{~b}$ MNs contribute a unique percentage of the total EPSP in a muscle-specific manner. Genetic ablation of 1s MNs (vCE and dCE) after innervation led to the expansion of $1 \mathrm{~b}$ NMJs and the elevation of their spontaneous release rates. Furthermore, some $1 \mathrm{~b}$ MNs elevated evoked neurotransmission while others remained unchanged. A recent study examining $\mathrm{m} 1$ found similar $1 \mathrm{~b}$ functional plasticity when $\mathrm{m} 1-1 \mathrm{~s}$ was ablated (Aponte-Santiago et al., 2020), but no structural compensation. This target specificity indicates heterogeneity in synaptic plasticity mechanisms. Silencing 1s MNs yielded partial m4-1b compensation, suggesting that 1s-evoked neurotransmission is an important factor in eliciting $1 \mathrm{~b}$ NMJ plasticity. In mutant larvae where the m41s NMJs never form, we observed no changes in m4-1b bouton number or spontaneous release rate, and decreased EPSP compensation. These results suggest that initial $1 \mathrm{~s}$ innervation may set up a reference point for robust $1 \mathrm{~b}$ synaptic plasticity.

Potential mechanisms to regulate $1 \mathrm{~b}$ synaptic plasticity A well established form of plasticity at the larval NMJ is synaptic homeostasis, which can manifest in presynaptic and/or postsynaptic changes, including neurotransmitter release (Petersen et al., 1997; Davis and Goodman, 1998; Davis, 2013) and neurotransmitter receptor dynamics and abundance (Turrigiano et al., 1998; Wierenga et al., 2005; Frank et al., 2020), respectively. Presynaptic homeostatic plasticity (PHP) maintains EPSPs at baseline when postsynaptic glutamate receptor function is perturbed (Davis and Müller, 2015; Frank et al., 2020) and can be induced in a synapse-specific manner (Newman et al., 2017; Li et al., 2018). In our study, the loss of 1s MNs could be interpreted as a decrease in glutamate receptor function by the muscle and trigger similar PHP mechanisms to induce compensatory changes at adjacent $1 \mathrm{~b}$ NMJs. Thus, further analysis of PHP pathways could reveal mechanisms underlying this convergent plasticity.

$1 \mathrm{~b}$ NMJs must adapt to accommodate the increased spontaneous and evoked neurotransmission. Our results do not reveal robust expansion of AZs or GluRs to support 1b synaptic plasticity, as observed in other studies where NMJ size is altered but total AZs or QCs remain the same (Goel et al., 2019a,b, 2020; Aponte-Santiago et al., 2020). Therefore, we propose that existing AZs may alter their properties. AZs exist in two different states, active or silent (Atwood and Wojtowicz, 1999; Ruiz et al., 2011), and a subset of AZs specializes in spontaneous release or evoked neurotransmission (Melom et al., 2013; Peled et al., 2014; Newman et al., 2017; Akbergenova et al., 2019). Thus, even without an increase in AZs, $1 \mathrm{~b}$ plasticity mechanisms may modify 
$\mathrm{AZ}$ properties to respond to the loss of $1 \mathrm{~s}$ inputs. Overall, our data suggest that silent AZs may become activated to increase the pools of spontaneous and evoked AZs as the ablation of $1 \mathrm{~s}$ MNs led to enhanced $1 \mathrm{~b}$ spontaneous release rates, target-specific compensation of EPSPs, and increased QC. Additionally, spontaneous and evoked activities may be independently regulated. Furthermore, the readily releasable pool size is under dynamic control during synaptic plasticity (Weyhersmüller et al., 2011; Müller et al., 2012) and could modulate m4-1b-evoked neurotransmitter release. Detailed examination of AZs will significantly bolster our understanding of the mechanism underlying 1b NMJ plasticity.

Prior studies reported that spontaneous neurotransmitter release regulates synaptic development in both mammals and Drosophila (Joseph et al., 2012; Choi et al., 2014; Andreae and Burrone, 2015, 2018; Cho et al., 2015; Kavalali, 2015). Thus, the expanded size of all 1b NMJs following $1 \mathrm{~s}$ ablation may be caused by the elevated spontaneous activity. These data also suggest that all $1 \mathrm{~b}$ MNs can detect and respond to the loss of adjacent $1 \mathrm{~s}$ inputs. However, the ability to differentially compensate the spontaneous and evoked activity is likely because of independent mechanisms since only some $1 \mathrm{~b}$ MNs elevate their EPSPs.

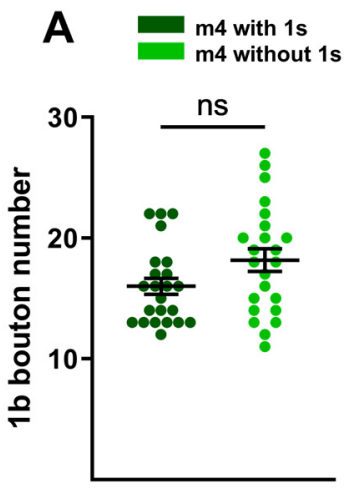

B

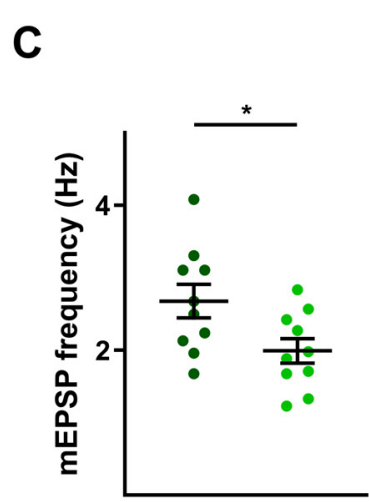

D

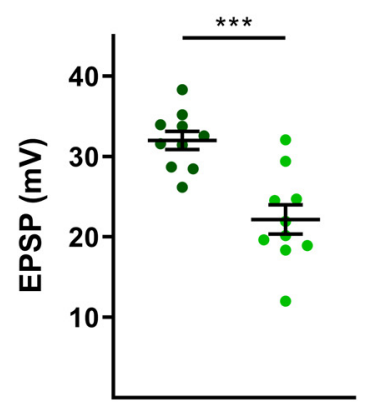

E

$\mathbf{F}$

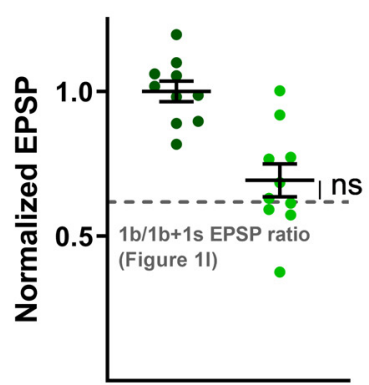

Figure 12. $\mathrm{m} 4 \mathrm{~s}$ that naturally lack $1 \mathrm{~s}$ innervation do not show $1 \mathrm{~b}$ plasticity. $\boldsymbol{A}$, Quantification of $1 \mathrm{~b}$ boutons from $\mathrm{m} 4$ with and without $1 \mathrm{~s}$ innervation $\left(t_{(44)}=1.885, p=0.0661\right.$, unpaired $t$ test). $\boldsymbol{B}$, Quantification of satellite boutons from $\mathrm{m} 4$ with and without $1 \mathrm{~s}$ innervation $\left(t_{(44)}=0.3895, p=0.6988\right.$, unpaired $t$ test). $C$, Quantification of mEPSP frequencies from $\mathrm{m} 4$ with and without $1 \mathrm{~s}$ innervation $\left(t_{(18)}=2.414, p=0.0267\right.$, unpaired $t$ test). D, Quantification of EPSP amplitudes from $\mathrm{m} 4$ with and without $1 \mathrm{~s}$ innervation $\left(t_{(18)}=4.576, p=0.0002\right.$, unpaired $t$ test). $\boldsymbol{E}$, Normalized EPSPs from $\mathrm{m} 4$ without $1 \mathrm{~s}$ innervation are compared with the EPSP ratio of $1 \mathrm{~b} / 1 \mathrm{~b}+1 \mathrm{~s}$ calculated from $\mathrm{m} 4$ in Figure 1 , indicated by the gray dashed line $\left(t_{(24)}=1.263, p=0.2186\right.$, unpaired $t$ test). $\boldsymbol{F}$, Normalized QC from $\mathrm{m} 4$ without $1 \mathrm{~s}$ innervation is compared with the $1 \mathrm{~b}$ baseline QC (see Materials and Methods; $t_{(24)}=0.1449, p=0.8860$, unpaired $t$ test). Error bars indicate \pm SEM. $* p<0.05$, $* * * p<0.001$, ns - not significant. $n$ values (NMJs/larva) are 23/17 and 23/17 for $\boldsymbol{A}$ and $\boldsymbol{B}$, and $10 / 7$ and $10 / 9$ for $\boldsymbol{C}-\boldsymbol{F}$.

\section{Correlation between synaptic weight and target-specific plasticity}

In complex neural circuits, dissecting contributions of individual inputs to the total postsynaptic activity, also referred to as synaptic weight (Magee and Cook, 2000; Bhalla, 2008), remains difficult because of thousands of converging inputs on a single cell. The larval NMJ facilitates the partitioning of synaptic inputs as each muscle is innervated by few MNs. In this study, we combined electrophysiology with calcium imaging and found that $1 \mathrm{~b}$ synaptic weights differ on $\mathrm{m} 6, \mathrm{~m} 12$, and $\mathrm{m} 4$. Together with the degree of EPSP compensation after the ablation of 1s MNs, there was a direct correlation with the level of target-specific synaptic weight. Thus, robust $1 \mathrm{~b}$ MNs that carry more synaptic drive may be endowed with certain synaptic plasticity mechanisms that respond to the loss of adjacent inputs. However, we cannot rule out regulatory roles for type II and type III MNs (Gorczyca et al., 1993; Chang and Keshishian, 1996; Koon et al., 2011) that are present on some muscles.

Interestingly, a similar correlation exists in Hebbian plasticity, where stronger synapses are more likely strengthened than weaker ones (Song et al., 2000; Babadi and Abbott, 2010). This correlation is also reflected in PHP. Two studies examined input-specific PHP on different muscles. On m4, PHP can be induced only at $1 \mathrm{~b}$ NMJs (Newman et al., 2017); however, on m6, PHP can be induced on both $1 \mathrm{~b}$ and 1s NMJs (Genc and Davis, 2019). This correlates with our observation that the $\mathrm{m} 4$ - $1 \mathrm{~b}$ has more synaptic weight than $\mathrm{m} 4$ $1 s$, whereas $\mathrm{m} 6-1 \mathrm{~b}$ and m6-1s have similar synaptic weights.
Together, homeostatic plasticity varies in target-specific and inputspecific manners, suggesting heterogeneous mechanisms.

\section{Establishing an EPSP set point for $1 \mathrm{~b}$ MN synaptic plasticity}

Models of synaptic homeostasis rely on an activity set point to stabilize neurons when confronted with perturbations (LeMasson et al., 1993; Liu et al., 1998; Turrigiano, 2007; Davis, 2013; O'Leary et al., 2014). Each target neuron must account for all presynaptic inputs to produce a defined output (i.e., the set point). The structural and functional properties of each input thus determine not only its contribution to the postsynaptic activity but also its ability to respond to perturbations in synaptic function. For example, transcription factors not only regulate the temporal expression of ion channels that shape neuronal excitability, but also homeostatic mechanisms (Turrigiano, 2007; Davis, 2013; Parrish et al., 2014; Engelmann and Haenold, 2016; Diering et al., 2017; Schaukowitch et al., 2017). Like many activity-dependent processes (Ataman et al., 2008; Carrillo et al., 2010; Berke et al., 2013; Vonhoff and Keshishian, 2017a), the optimal set point may be established during a narrow time window of development. This hypothesis was tested in a Drosophila seizure mutant by inhibiting activity during embryonic development and observing the suppression of seizures in postembryonic stages (Giachello and Baines, 2015). Thus, manipulating activity during an embryonic critical period may alter the activity set point. 
In this study, one intriguing hypothesis is that $1 \mathrm{~b}+1 \mathrm{~s}$ coinnervation determines the EPSP set point during embryogenesis and is referenced by some $1 \mathrm{~b}$ NMJs to compensate for the loss of 1s MNs. We propose a model to describe how $1 \mathrm{~b}$ NMJs increase their sizes and spontaneous and evoked neurotransmission because of the loss of convergent $1 \mathrm{~s}$ MNs. The neuromuscular innervation map is formed during late embryonic development (Prokop et al., 1996; Yoshihara et al., 1997; Vonhoff and Keshishian, 2017b). Since the maximum EPSP amplitude is established at the first instar stage and maintained throughout larval development (Davis and Goodman, 1998; Li et al., 2002), the set point is likely determined by the initial $1 \mathrm{~b}+1 \mathrm{~s}$ coinnervation. Blocking the initial formation of 1s NMJs would create a set point that is devoid of $1 \mathrm{~s}$ influence; and thus, the corresponding $1 \mathrm{~b}$ NMJs would not compensate toward the wild-type EPSP but instead to this alternate EPSP set point. If the $1 \mathrm{~s} \mathrm{MN}$ is ablated after synaptogenesis, the correct set point is established and the $1 \mathrm{~b}$ responds accordingly. Removing $1 \mathrm{~s}$ innervation at various time points during larval development will provide a deeper understanding of the temporal dynamics of $1 \mathrm{~b}$ plasticity and whether the EPSP set point is maintained by $1 b+1$ s activities.

\section{Synaptic plasticity in other convergent neural circuits}

The Drosophila neuromuscular circuit is a reductionist system with polyinnervated muscles. The loss of an input triggering a compensatory increase in synaptic growth or function of a convergent input could be viewed as synaptic competition. Synaptic competition is well characterized at the vertebrate neuromuscular junction, where neonatal muscles are innervated by several motor axons, but only one survives to adulthood (Sanes and Lichtman, 1999; Tapia et al., 2012). Neuromuscular activity is critical for this pruning process but the signaling pathway is not completely understood (Schuldiner and Yaron, 2015; Lee, 2020). Studying convergent synaptic plasticity at the Drosophila NMJ may shed light on this process.

Mechanisms uncovered at the NMJ can act in more complex circuits in vertebrates and invertebrates. For example, in the fly $\mathrm{VNC}$, each $\mathrm{MN}$ receives sensory information from many interneurons to regulate motor behaviors (Heckscher et al., 2015; Schneider-Mizell et al., 2016; Kohsaka et al., 2019). The convergence of inputs on one MN may establish a homeostatic set point in the MN, similar to the muscle. The complex interneuron-MN connectivity more closely resembles that of vertebrate CNS neurons with polysynaptic dendrites (Kim et al., 2009; Heckscher et al., 2015; Kohsaka et al., 2019; Zarin et al., 2019). Additionally, age-related (Bergado and Almaguer, 2002; Mattson and Magnus, 2006; Mostany et al., 2013; Griffith et al., 2014; Petralia et al., 2014) and disease-related (Gorman, 2008; Milnerwood and Raymond, 2010; Lepeta et al., 2016; Salvadores et al., 2017; SmithDijak et al., 2019) changes in synaptic function and cell survival have been observed in CNS and neuromuscular circuits. In patients and animal models of Alzheimer's disease, while some neurons are depressed in amyloid- $\beta$ plaque-enriched regions, other neurons show a compensatory hyperactivation (Busche et al., 2012; Merlo et al., 2019). Thus, future studies at the NMJ and other circuits will elucidate the mechanisms governing how and when the activity set point is defined in a target-specific manner and how neurons respond to dysfunctional neighbors.

\section{References}

Akbergenova Y, Cunningham KL, Zhang YV, Weiss S, Littleton JT (2018) Characterization of developmental and molecular factors underlying release heterogeneity at Drosophila synapses. eLife 7:e38268.
Andreae LC, Burrone J (2015) Spontaneous neurotransmitter release shapes dendritic arbors via long-range activation of NMDA receptors. Cell Rep 10:873-882.

Andreae LC, Burrone J (2018) The role of spontaneous neurotransmission in synapse and circuit development. J Neurosci Res 96:354-359.

Aponte-Santiago NA, Ormerod KG, Akbergenova Y, Littleton JT (2020) Synaptic plasticity induced by differential manipulation of tonic and phasic motoneurons in Drosophila. J Neurosci 40:6270-6288.

Ashley J, Sorrentino V, Lobb-Rabe M, Nagarkar-Jaiswal S, Tan L, Xu S, Xiao Q, Zinn K, Carrillo RA (2019) Transsynaptic interactions between IgSF proteins DIP- $\alpha$ and Dpr10 are required for motor neuron targeting specificity. Elife 8:e42690.

Ataman B, Ashley J, Gorczyca M, Ramachandran P, Fouquet W, Sigrist SJ, Budnik V (2008) Rapid activity-dependent modifications in synaptic structure and function require bidirectional Wnt signaling. Neuron 57:705-718.

Atwood HL, Wojtowicz JM (1999) Silent synapses in neural plasticity: current evidence. Learn Mem 6:542-571.

Babadi B, Abbott LF (2010) Intrinsic stability of temporally shifted spike-timing dependent plasticity. PLOS Comput Biol 6:e1000961.

Bergado JA, Almaguer W (2002) Aging and synaptic plasticity: a review. Neural Plast 9:217-232.

Berke B, Wittnam J, McNeill E, Van Vactor DL, Keshishian H (2013) Retrograde BMP signaling at the synapse: a permissive signal for synapse maturation and activity-dependent plasticity. J Neurosci 33:17937-17950.

Berry KP, Nedivi E (2017) Spine dynamics: are they all the same? Neuron 96:43-55.

Bhalla US (2008) How to record a million synaptic weights in a hippocampal slice. PLoS Comput Biol 4:e1000098.

Bownes M (1975) A photographic study of development in the living embryo of Drosophila melanogaster. J Embryol Exp Morphol 33:789-801.

Broadie KS, Bate M (1993) Development of the embryonic neuromuscular synapse of Drosophila melanogaster. J Neurosci 13:144-166.

Broadus J, Skeath JB, Spana EP, Bossing T, Technau G, Doe CQ (1995) New neuroblast markers and the origin of the aCC/pCC neurons in the Drosophila central nervous system. Mech Dev 53:393-402.

Budnik V, Zhong Y, Wu CF (1990) Morphological plasticity of motor axons in Drosophila mutants with altered excitability. J Neurosci 10:3754-3768.

Busche MA, Chen X, Henning HA, Reichwald J, Staufenbiel M, Sakmann B, Konnerth A (2012) Critical role of soluble amyloid- $\beta$ for early hippocampal hyperactivity in a mouse model of Alzheimer's disease. Proc Natl Acad Sci U S A 109:8740-8745.

Carrillo RA, Olsen DP, Yoon KS, Keshishian H (2010) Presynaptic activity and CaMKII modulate retrograde semaphorin signaling and synaptic refinement. Neuron 68:32-44.

Carrillo RA, Özkan E, Menon KP, Nagarkar-Jaiswal S, Lee PT, Jeon M, Birnbaum ME, Bellen HJ, Garcia KC, Zinn K (2015) Control of synaptic connectivity by a network of Drosophila IgSF cell surface proteins. Cell 163:1770-1782.

Chang TN, Keshishian H (1996) Laser ablation of Drosophila embryonic motoneurons causes ectopic innervation of target muscle fibers. J Neurosci 16:5715-5726.

Chistiakova M, Ilin V, Roshchin M, Bannon N, Malyshev A, Kisvárday Z, Volgushev M (2019) Distinct heterosynaptic plasticity in fast spiking and non-fast-spiking inhibitory neurons in rat visual cortex. J Neurosci 39:6865-6878.

Cho RW, Buhl LK, Volfson D, Tran A, Li F, Akbergenova Y, Littleton JT (2015) Phosphorylation of complexin by PKA regulates activity-dependent spontaneous neurotransmitter release and structural synaptic plasticity. Neuron 88:749-761.

Choi BJ, Imlach WL, Jiao W, Wolfram V, Wu Y, Grbic M, Cela C, Baines RA, Nitabach MN, McCabe BD (2014) Miniature neurotransmission regulates Drosophila synaptic structural maturation. Neuron 82:618-634.

Choi JC, Park D, Griffith LC (2004) Electrophysiological and morphological characterization of identified motor neurons in the Drosophila third instar larva central nervous system. J Neurophysiol 91:2353-2365.

Cluskey S, Ramsden DB (2001) Mechanisms of neurodegeneration in amyotrophic lateral sclerosis. Mol Pathol 54:386-392.

Collins CA, DiAntonio A (2007) Synaptic development: insights from Drosophila. Curr Opin Neurobiol 17:35-42.

Davis GW (2013) Homeostatic signaling and the stabilization of neural function. Neuron 80:718-728. 
Davis GW, Goodman CS (1998) Genetic analysis of synaptic development and plasticity: homeostatic regulation of synaptic efficacy. Curr Opin Neurobiol 8:149-156.

Davis GW, Müller M (2015) Homeostatic control of presynaptic neurotransmitter release. Annu Rev Physiol 77:251-270.

Dickman DK, Lu Z, Meinertzhagen IA, Schwarz TL (2006) Altered synaptic development and active zone spacing in endocytosis mutants. Curr Biol 16:591-598.

Diering GH, Nirujogi RS, Roth RH, Worley PF, Pandey A, Huganir RL (2017) Homerla drives homeostatic scaling-down of excitatory synapses during sleep. Science 355:511-515.

Doe CQ, Smouse D, Goodman CS (1988) Control of neuronal fate by the Drosophila segmentation gene even-skipped. Nature 333:376-378.

Engelmann C, Haenold R (2016) Transcriptional control of synaptic plasticity by transcription factor NF-kappaB. Neural Plast 2016:7027949.

Feeney CJ, Karunanithi S, Pearce J, Govind CK, Atwood HL (1998) Motor nerve terminals on abdominal muscles in larval flesh flies, Sarcophaga bullata: comparisons with Drosophila. J Comp Neurol 402:197-209.

Frank CA, James TD, Müller M (2020) Homeostatic control of Drosophila neuromuscular junction function. Synapse 74:e22133.

Genc O, Davis GW (2019) Target-wide induction and synapse type-specific robustness of presynaptic homeostasis. Curr Biol 29:3863-3873.e2.

Giachello CN, Baines RA (2015) Inappropriate neural activity during a sensitive period in embryogenesis results in persistent seizure-like behavior. Curr Biol 25:2964-2968.

Goel P, Dufour Bergeron D, Böhme MA, Nunnelly L, Lehmann M, Buser C, Walter AM, Sigrist SJ, Dickman D (2019a) Homeostatic scaling of active zone scaffolds maintains global synaptic strength. J Cell Biol 218:1706-1724.

Goel P, Khan M, Howard S, Kim G, Kiragasi B, Kikuma K, Dickman D (2019b) A screen for synaptic growth mutants reveals mechanisms that stabilize synaptic strength. J Neurosci 39:4051-4065.

Goel P, Nishimura S, Chetlapalli K, Li X, Chen C, Dickman D (2020) Distinct target-specific mechanisms homeostatically stabilize transmission at preand post-synaptic compartments. Front Cell Neurosci 14:196.

Gorczyca M, Augart C, Budnik V (1993) Insulin-like receptor and insulinlike peptide are localized at neuromuscular junctions in Drosophila. J Neurosci 13:3692-3704.

Gorman AM (2008) Neuronal cell death in neurodegenerative diseases: recurring themes around protein handling. J Cell Mol Med 12:2263-2280.

Griffith WH, Dubois DW, Fincher A, Peebles KA, Bizon JL, Murchison D (2014) Characterization of age-related changes in synaptic transmission onto F344 rat basal forebrain cholinergic neurons using a reduced synaptic preparation. J Neurophysiol 111:273-286.

Guan B, Hartmann B, Kho YH, Gorczyca M, Budnik V (1996) The Drosophila tumor suppressor gene, $\mathrm{dlg}$, is involved in structural plasticity at a glutamatergic synapse. Curr Biol 6:695-706.

Hallermann S, Heckmann M, Kittel RJ (2010) Mechanisms of short-term plasticity at neuromuscular active zones of Drosophila. HFSP J 4:72-84.

Halpern ME, Chiba A, Johansen J, Keshishian H (1991) Growth cone behavior underlying the development of stereotypic synaptic connections in Drosophila embryos. J Neurosci 11:3227-3238.

Han TH, Vicidomini R, Ramos CI, Wang Q, Nguyen P, Jarnik M, Lee C-H, Stawarski M, Hernandez RX, Macleod GT, Serpe M (2020) Neto- $\alpha$ controls synapse organization and homeostasis at the drosophila neuromuscular junction. Cell Rep 32:107866.

Hartenstein V, Rudloff E, Campos-Ortega JA (1987) The pattern of proliferation of the neuroblasts in the wild-type embryo of Drosophila melanogaster. Rouxs Arch Dev Biol 196:473-485.

Heckscher ES, Zarin AA, Faumont S, Clark MQ, Manning L, Fushiki A, Schneider-Mizell CM, Fetter RD, Truman JW, Zwart MF, Landgraf M, Cardona A, Lockery SR, Doe CQ (2015) Even-Skipped(+) interneurons are core components of a sensorimotor circuit that maintains left-right symmetric muscle contraction amplitude. Neuron 88:314-329.

Hedrick NG, Harward SC, Hall CE, Murakoshi H, McNamara JO, Yasuda R (2016) Rho GTPase complementation underlies BDNF-dependent homo- and heterosynaptic plasticity. Nature 538:104-108.

Hoang B, Chiba A (2001) Single-cell analysis of Drosophila larval neuromuscular synapses. Dev Biol 229:55-70.

Jan LY, Jan YN (1976) Properties of the larval neuromuscular junction in Drosophila melanogaster. J Physiol 262:189-214.

Jarecki J, Keshishian H (1995) Role of neural activity during synaptogenesis in Drosophila. J Neurosci 15:8177-8190.
Joseph J, Dunn FA, Stopfer M (2012) Spontaneous olfactory receptor neuron activity determines follower cell response properties. J Neurosci 32:2900-2910.

Jungenitz $\mathrm{T}$, Beining $\mathrm{M}$, Radic $\mathrm{T}$, Deller $\mathrm{T}$, Cuntz $\mathrm{H}$, Jedlicka $\mathrm{P}$, Schwarzacher SW (2018) Structural homo- and heterosynaptic plasticity in mature and adult newborn rat hippocampal granule cells. Proc Natl Acad Sci U S A 115:E4670-E4679.

Kallmünzer B, Sörensen B, Neuhuber WL, Wörl J (2008) Enteric co-innervation of striated muscle fibres in human oesophagus. Neurogastroenterol Motil 20:597-610.

Kavalali ET (2015) The mechanisms and functions of spontaneous neurotransmitter release. Nat Rev Neurosci 16:5-16.

Kim MD, Wen Y, Jan YN (2009) Patterning and organization of motor neuron dendrites in the Drosophila larva. Dev Biol 336:213-221.

Kittel RJ, Wichmann C, Rasse TM, Fouquet W, Schmidt M, Schmid A, Wagh DA, Pawlu C, Kellner RR, Willig KI, Hell SW, Buchner E, Heckmann M, Sigrist SJ (2006) Bruchpilot promotes active zone assembly, Ca2 + channel clustering, and vesicle release. Science 312:1051-1054.

Kohsaka H, Zwart MF, Fushiki A, Fetter RD, Truman JW, Cardona A, Nose A (2019) Regulation of forward and backward locomotion through intersegmental feedback circuits in Drosophila larvae. Nat Commun 10:2654.

Koh YH, Popova E, Thomas U, Griffith LC, Budnik V (1999) Regulation of DLG localization at synapses by CaMKII-dependent phosphorylation. Cell 98:353-363.

Koon AC, Ashley J, Barria R, DasGupta S, Brain R, Waddell S, Alkema MJ, Budnik V (2011) Autoregulatory and paracrine control of synaptic and behavioral plasticity by octopaminergic signaling. Nat Neurosci 14:190-199.

Kruijssen DLH, Wierenga CJ (2019) Single synapse LTP: a matter of context? Front Cell Neurosci 13:496.

Kurdyak P, Atwood HL, Stewart BA, Wu CF (1994) Differential physiology and morphology of motor axons to ventral longitudinal muscles in larval Drosophila. J Comp Neurol 350:463-472.

Landgraf M, Bossing T, Technau GM, Bate M (1997) The origin, location, and projections of the embryonic abdominal motorneurons of Drosophila. J Neurosci 17:9642-9655.

Lee HK, Wright AP, Zinn K (2009) Live dissection of Drosophila embryos: streamlined methods for screening mutant collections by antibody staining. J Vis $\operatorname{Exp}(34): 1647$

Lee J, Wu CF (2010) Orchestration of stepwise synaptic growth by $\mathrm{K}^{+}$and $\mathrm{Ca}^{2+}$ channels in Drosophila. J Neurosci 30:15821-15833.

Lee YI (2020) Developmental neuromuscular synapse elimination: activitydependence and potential downstream effector mechanisms. Neurosci Lett 718:134724.

LeMasson G, Marder E, Abbott LF (1993) Activity-dependent regulation of conductances in model neurons. Science 259:1915-1917.

Lepeta K, Lourenco MV, Schweitzer BC, Martino Adami PV, Banerjee P, Catuara-Solarz S, de La Fuente Revenga M, Guillem AM, Haidar M, Ijomone OM, Nadorp B, Qi L, Perera ND, Refsgaard LK, Reid KM, Sabbar M, Sahoo A, Schaefer N, Sheean RK, Suska A, et al (2016) Synaptopathies: synaptic dysfunction in neurological disorders-a review from students to students. J Neurochem 138:785-805.

Li H, Peng X, Cooper RL (2002) Development of Drosophila larval neuromuscular junctions: maintaining synaptic strength. Neuroscience 115: 505-513.

Li X, Goel P, Chen C, Angajala V, Chen X, Dickman DK (2018) Synapse-specific and compartmentalized expression of presynaptic homeostatic potentiation. Elife 7:e34338

Liu Z, Golowasch J, Marder E, Abbott LF (1998) A model neuron with activity-dependent conductances regulated by multiple calcium sensors. J Neurosci 18:2309-2320.

Lnenicka GA, Keshishian H (2000) Identified motor terminals in Drosophila larvae show distinct differences in morphology and physiology. J Neurobiol 43:186-197.

Magee JC, Cook EP (2000) Somatic EPSP amplitude is independent of synapse location in hippocampal pyramidal neurons. Nat Neurosci 3:895-903.

Martin AR (1955) A further study of the statistical composition on the endplate potential. J Physiol 130:114-122.

Mattson MP, Magnus T (2006) Ageing and neuronal vulnerability. Nat Rev Neurosci 7:278-294.

Megías M, Emri Z, Freund TF, Gulyás AI (2001) Total number and distribution of inhibitory and excitatory synapses on hippocampal CA1 pyramidal cells. Neuroscience 102:527-540. 
Melom JE, Akbergenova Y, Gavornik JP, Littleton JT (2013) Spontaneous and evoked release are independently regulated at individual active zones. J Neurosci 33:17253-17263.

Meng JL, Wang Y, Carrillo RA, Heckscher ES (2020) Temporal transcription factors determine circuit membership by permanently altering motor neuron-to-muscle synaptic partnerships. Elife 9:e56898.

Menon KP, Carrillo RA, Zinn K (2013) Development and plasticity of the Drosophila larval neuromuscular junction. Wiley Interdiscip Rev Dev Biol 2:647-670.

Merlo S, Spampinato SF, Sortino MA (2019) Early compensatory responses against neuronal injury: a new therapeutic window of opportunity for Alzheimer's Disease? CNS Neurosci Ther 25:5-13.

Milnerwood AJ, Raymond LA (2010) Early synaptic pathophysiology in neurodegeneration: insights from Huntington's disease. Trends Neurosci 33:513-523.

Mostany R, Anstey JE, Crump KL, Maco B, Knott G, Portera-Cailliau C (2013) Altered synaptic dynamics during normal brain aging. J Neurosci 33:4094-4104.

Müller M, Liu KS, Sigrist SJ, Davis GW (2012) RIM controls homeostatic plasticity through modulation of the readily-releasable vesicle pool. J Neurosci 32:16574-16585.

Neuhuber WL, Wörl J (2016) Enteric co-innervation of striated muscle in the esophagus: still enigmatic? Histochem Cell Biol 146:721-735.

Newman ZL, Hoagland A, Aghi K, Worden K, Levy SL, Son JH, Lee LP, Isacoff EY (2017) Input-specific plasticity and homeostasis at the Drosophila larval neuromuscular junction. Neuron 93:1388-1404.e10.

Nguyen CT, Stewart BA (2016) The influence of postsynaptic structure on missing quanta at the Drosophila neuromuscular junction. BMC Neurosci 17:53.

Nose A (2012) Generation of neuromuscular specificity in Drosophila: novel mechanisms revealed by new technologies. Front Mol Neurosci 5:62.

O'Connor-Giles KM, Ganetzky B (2008) Satellite signaling at synapses. Fly (Austin) 2:259-261.

O'Connor-Giles KM, Ho LL, Ganetzky B (2008) Nervous wreck interacts with thickveins and the endocytic machinery to attenuate retrograde BMP signaling during synaptic growth. Neuron 58:507-518.

O'Leary T, Williams AH, Franci A, Marder E (2014) Cell types, network homeostasis, and pathological compensation from a biologically plausible ion channel expression model. Neuron 82:809-821.

Oh WC, Parajuli LK, Zito K (2015) Heterosynaptic structural plasticity on local dendritic segments of hippocampal CA1 neurons. Cell Rep 10:162169.

Parrish JZ, Kim CC, Tang L, Bergquist S, Wang T, Derisi JL, Jan LY, Jan YN, Davis GW (2014) Krüppel mediates the selective rebalancing of ion channel expression. Neuron 82:537-544.

Pauls D, von Essen A, Lyutova R, van Giesen L, Rosner R, Wegener C, Sprecher SG (2015) Potency of transgenic effectors for neurogenetic manipulation in Drosophila larvae. Genetics 199:25-37.

Peled ES, Newman ZL, Isacoff EY (2014) Evoked and spontaneous transmission favored by distinct sets of synapses. Curr Biol 24:484-493.

Perry S, Goel P, Tran NL, Pinales C, Buser C, Miller DL, Ganetzky B, Dickman D (2020) Developmental arrest of Drosophila larvae elicits presynaptic depression and enables prolonged studies of neurodegeneration. Development 147:dev186312.

Petersen SA, Fetter RD, Noordermeer JN, Goodman CS, DiAntonio A (1997) Genetic analysis of glutamate receptors in Drosophila reveals a retrograde signal regulating presynaptic transmitter release. Neuron 19:1237-1248

Petralia RS, Mattson MP, Yao PJ (2014) Communication breakdown: the impact of ageing on synapse structure. Ageing Res Rev 14:31-42.

Prokop A, Landgraf M, Rushton E, Broadie K, Bate M (1996) Presynaptic development at the Drosophila neuromuscular junction: assembly and localization of presynaptic active zones. Neuron 17:617-626.

Ruiz R, Cano R, Casañas JJ, Gaffield MA, Betz WJ, Tabares L (2011) Active zones and the readily releasable pool of synaptic vesicles at the neuromuscular junction of the mouse. J Neurosci 31:2000-2008.

Salvadores N, Sanhueza M, Manque P, Court FA (2017) Axonal degeneration during aging and its functional role in neurodegenerative disorders. Front Neurosci 11:451.
Sanes JR, Lichtman JW (1999) Development of the vertebrate neuromuscular junction. Annu Rev Neurosci 22:389-442.

Schaefer JE, Worrell JW, Levine RB (2010) Role of intrinsic properties in Drosophila motoneuron recruitment during fictive crawling. J Neurophysiol 104:1257-1266.

Schaukowitch K, Reese AL, Kim SK, Kilaru G, Joo JY, Kavalali ET, Kim TK (2017) An intrinsic transcriptional program underlying synaptic scaling during activity suppression. Cell Rep 18:1512-1526.

Schneider-Mizell CM, Gerhard S, Longair M, Kazimiers T, Li F, Zwart MF, Champion A, Midgley FM, Fetter RD, Saalfeld S, Cardona A (2016) Quantitative neuroanatomy for connectomics in Drosophila. Elife 5: e12059.

Schuldiner O, Yaron A (2015) Mechanisms of developmental neurite pruning. Cell Mol Life Sci 72:101-119.

Sigrist SJ, Reiff DF, Thiel PR, Steinert JR, Schuster CM (2003) Experience-dependent strengthening of Drosophila neuromuscular junctions. J Neurosci 23:6546-6556.

Smith-Dijak AI, Sepers MD, Raymond LA (2019) Alterations in synaptic function and plasticity in Huntington disease. J Neurochem 150:346-365.

Song S, Miller KD, Abbott LF (2000) Competitive Hebbian learning through spike-timing-dependent synaptic plasticity. Nat Neurosci 3:919-926.

Sweeney ST, Broadie K, Keane J, Niemann H, O’Kane CJ (1995) Targeted expression of tetanus toxin light chain in Drosophila specifically eliminates synaptic transmission and causes behavioral defects. Neuron 14:341-351.

Tapia JC, Wylie JD, Kasthuri N, Hayworth KJ, Schalek R, Berger DR, Guatimosim C, Seung HS, Lichtman JW (2012) Pervasive synaptic branch removal in the mammalian neuromuscular system at birth. Neuron 74:816-829.

Torroja L, Packard M, Gorczyca M, White K, Budnik V (1999) The Drosophila $\beta$-amyloid precursor protein homolog promotes synapse differentiation at the neuromuscular junction. J Neurosci 19:7793-7803.

Turrigiano G (2007) Homeostatic signaling: the positive side of negative feedback. Curr Opin Neurobiol 17:318-324.

Turrigiano GG, Leslie KR, Desai NS, Rutherford LC, Nelson SB (1998) Activity-dependent scaling of quantal amplitude in neocortical neurons. Nature 391:892-896.

Van Vactor D, Sigrist SJ (2017) Presynaptic morphogenesis, active zone organization and structural plasticity in Drosophila. Curr Opin Neurobiol 43:119-129.

Venkatasubramanian L, Guo Z, Xu S, Tan L, Xiao Q, Nagarkar-Jaiswal S, Mann RS (2019) Stereotyped terminal axon branching of leg motor neurons mediated by IgSF proteins DIP- $\alpha$ and Dpr10. Elife 8:e42692.

Vonhoff F, Keshishian H (2017a) Activity-dependent synaptic refinement: new insights from Drosophila. Front Syst Neurosci 11:23.

Vonhoff F, Keshishian H (2017b) In vivo calcium signaling during synaptic refinement at the Drosophila neuromuscular junction. J Neurosci 37:5511-5526.

Weyhersmüller A, Hallermann S, Wagner N, Eilers J (2011) Rapid active zone remodeling during synaptic plasticity. J Neurosci 31:6041-6052.

Wierenga CJ, Ibata K, Turrigiano GG (2005) Postsynaptic expression of homeostatic plasticity at neocortical synapses. J Neurosci 25:2895-2905.

Xu S, Xiao Q, Cosmanescu F, Sergeeva AP, Yoo J, Lin Y, Katsamba PS, Ahlsen G, Kaufman J, Linaval NT, Lee PT, Bellen HJ, Shapiro L, Honig B, Tan L, Zipursky SL (2018) Interactions between the Ig-Superfamily Proteins DIP-alpha and Dpr6/10 Regulate Assembly of Neural Circuits. Neuron 100:1369-1384 e1366.

Yoshihara M, Rheuben MB, Kidokoro Y (1997) Transition from growth cone to functional motor nerve terminal in Drosophila embryos. J Neurosci $17: 8408-8426$

Zarin AA, Mark B, Cardona A, Litwin-Kumar A, Doe CQ (2019) A multilayer circuit architecture for the generation of distinct locomotor behaviors in Drosophila. Elife 8:e51781.

Zhou L, Schnitzler A, Agapite J, Schwartz LM, Steller H, Nambu JR (1997) Cooperative functions of the reaper and head involution defective genes in the programmed cell death of Drosophila central nervous system midline cells. Proc Natl Acad Sci U S A 94:5131-5136. 